\title{
Vibro-acoustic optimization Study for the Volute Casing of a
}

\section{Centrifugal Fan}

\author{
Jianhua Zhang ${ }^{1}$, Wuli Chu ${ }^{2,3}$, Jinghui Zhang ${ }^{1}, Y_{i}$ Lv $^{1}$ \\ 1. School of Aircraft, Xi'an Aeronautical University, Xi'an, Shaanxi, China, 710072 \\ 2. School of Power \& Energy, Northwestern Polytechnical University, Xi'an, Shaanxi, China, 710072 \\ 3. Collaborative Innovation Center of Advanced Aero-Engine, Beijing, China, 100191 \\ corresponding author: lieying.2004@163.com
}

\begin{abstract}
Concerning fan systems with an air pipe connecting air intake and a closed outlet, aerodynamic noise cannot be directly transmitted from the fan inlet and outlet to the outside. At this moment, the volute vibrational radiation noise induced casing surface vibration is the major noise component. The main factors affecting the fan vibrational noise are analyzed through theoretical derivation, then a vibrational noise optimization control method for the volute casing is proposed that considered the influence of vibro-acoustic coupling, taking the panel thickness of the volute (front-panel thickness [FT], side-panel thickness [ST], and back-panel thickness [BT]) as design variables, and the acoustical power of the volute surface and the total mass of the volute as the optimal target function. The optimization method is mainly divided into three main parts: the first was based on the simulation of unsteady flow of the fan to obtain the vibrational noise source; the second, using the design of experimental (DOE) method and the proposed numerical simulation of fluid-structure-acoustic coupling method to obtain the designing space, then the radical-based function (RBF) method is used to construct the approximate surrogate model instead of the simulation model previously mentioned, which was used to provide the basic mathematical model for the optimization of the next part; the third part, implementing the low vibrational noise optimization for the fan volute, applied the single-target (taking volute radiated acoustical power as the target function) and the multi-target (taking the volute radiated acoustical power and volute total mass as the target function) methods. In addition, the fan aerodynamic performance, volute casing surface fluctuations, and vibration response were validated by experiments, showing good agreement. It is of utmost importance that the dynamic pressure measurements and vibrational tests on the volute casing verify the accuracy of the numerical calculation. The optimization results showed that the vibrational noise optimization method proposed in this study can effectively reduce the vibration noise of the fan, obtaining a maximum value of noise reduction of $7.3 \mathrm{~dB}$. The optimization identified in this paper provides a significant reference for the design of a low-vibrational-noise volute.
\end{abstract}

Key words: Centrifugal fan; Unsteady flow; Vibroacoustics; fluid-structure-acoustic coupling; Optimization

\section{Introduction}

Centrifugal fan is considered as a common turbomachinery, which are widely used in ventilation systems of the ship cabin and other sites, bringing comfortable working and living environments for people. However, the noise and vibrations generated with the fan running troubled us, thus study the mechanism of noise and vibration generation and propagation became more and more important. In some particular application environments, unsteady flow-induced vibrational noise is equally important. Aerodynamic-noise-induced unsteady flow of a fan cannot directly spread to the outside when the fan system inlet and outlet are entirely connected to the extended pipe. At this moment, the fan casing and the inlet and outlet pipe vibration noise caused by the vibrations of the volute surface are predominant. Therefore, an intensive study of the generation mechanism of the vibrational noise and the noise reduction method is necessary. In fact, the fan noise induced by unsteady flow belongs to fluidstructure coupling noise, and the impeller and volute can be classified as an elastomer; in particular, the volute vibration cannot be neglected in large fans [1]. There are few studies on the vibration noise induced by the vibration of the casing in a centrifugal fan. However, this type of noise is prominent in large-scale fan systems and fans with closed pipelines. At present, research on vibration noise induced by casing vibrations resulting from impeller outlet unsteady flow is usually produced using simulation methods. A prediction method based on a method of combining boundary element method (BEM) calculations with experimental measurement was proposed by Koopmann [2]. In this method, the aerodynamic noise is isolated, and the volute vibrations induced by the unsteady flow are calculated separately, and the pressure fluctuations 
required for noise and vibration calculations are obtained experimentally. On this basis, some scholars such as Hwang [3], Cai [4-5] and $\mathrm{Lu} \mathrm{[6]} \mathrm{have} \mathrm{used} \mathrm{the} \mathrm{same} \mathrm{method} \mathrm{to} \mathrm{calculate} \mathrm{the} \mathrm{vibrational} \mathrm{sound} \mathrm{radiation} \mathrm{of} \mathrm{a} \mathrm{compressor} \mathrm{and} \mathrm{the} \mathrm{T9-19}$ No.4 industrial centrifugal fan. Cai Jiancheng [7] calculated the vibrational sound radiation of a volute casing of the same centrifugal fan using a fluid-structure-acoustic coupling method. These research studies have been of benefit in promoting the development of research of vibrational sound radiation on volute casings, allowing for a deeper understanding of vibrational noise during the centrifugal fan operation, and have provided a useful reference for noise reduction of such turbomachinery. The purpose of vibrational noise research is to explore the generation mechanism of vibrational noise, then propose targeted methods of vibration and noise reduction. Concerning vibration and noise control, there are certain means: controlling the vibrational source, such as vibration absorption and vibration isolation [8]; dynamic vibration absorption; damping vibration control [9]; and structural vibration control [10-20]. At present, a structural vibration control method that meets specific requirements by modifying the dynamic characteristics of the controlled object without adding any subsystem is a research hotspot. Moreover, current structural vibration control is focused on structural optimization. However, a centrifugal fan casing belongs to a thin-casing structure, and the vibrational sound power of the thin-casing is a quadratic function of the structural vibration velocity [10-12]. As optimization reduces the structural vibration speed of a fan casing, it can be concluded that the sound power radiation must be reduced within a specific range. The optimal design of a thin-casing structure usually uses the panel thickness as the design variable and the square sum of the vibration velocities of the nodes on the wall as the optimization target function [13-14]. Adopting the mentioned method, Zhou et al. [15] and Lu et al. [16] implemented the optimization study on structure vibration control and noise reduction for the T9-19 No.4A centrifugal fan.

By reducing fan casing vibration, an effect of casing noise reduction is achieved using the aforementioned optimization method, which sets the vibration (node vibrational speed) as the target function. However, this method does not consider the propagation of sound waves and sound boundary influences on the calculation results; thus, deviation is nearly inevitable. The integration of structural-acoustic optimization correctly eliminates these drawbacks. This method has been used in the automotive field and shows that sound radiation generated by the excitation of body surface vibrations on the engine is substantially reduced [17-20] after optimization. Based on the aforementioned advantages of optimization, the author proposed a vibration-acoustic integrated optimization design method that is suitable for turbomachinery volute. In investigating the vibrational noise of the studied marine centrifugal fans, the following two aspects were a focus:

1) In this study, we proposed a numerical method for the unidirectional coupling of a flow field-structure-sound field. The rationality of this single coupling is verified by a volute wall vibration test.

2) To analyze the influence factors of vibrational noise and reduce the vibrational sound radiation induced by unsteady flow in the fan, detailed theoretical derivation of vibration noise is put forward

3) To control the vibrational noise of a certain type of marine centrifugal fan volute, an optimization method considering the influence of vibro-acoustic coupling is proposed. Under the premise that whether there exists volute total mass constraints, accordingly taking the panel thickness of the volute casing (FT, ST, BT) as the design variable, this study conducted the low vibrational noise single target (taking the volute vibrational radiated sound power as the target function) and multi-target optimization (taking the volute vibrational radiated sound power and total mass as the target function).

\section{NOMENCLATURE}

$\mathrm{B}=$ volute width $(\mathrm{mm})$

$\mathrm{D}_{2}=$ impeller outlet diameter $(\mathrm{m})$

$\mathrm{Q}=$ flow rate $\left(\mathrm{m}^{3} \mathrm{~s}-1\right)$

$\mathrm{Z}=$ the axial distance from the monitoring point to the volute rear casing $(\mathrm{mm})$

$\mathrm{BEP}=$ the best design point

$\mathrm{BPF}=$ the fundamental frequency, the blade passing frequency $(\mathrm{Hz})$

$\mathrm{c}=$ speed of sound (m s-1)

$\mathrm{u}_{2}=$ circumferential velocity of impeller outlet ( $\left.\mathrm{m} \mathrm{s}-1\right)$

$\mathrm{P}_{\mathrm{T}}=$ total pressure rise $(\mathrm{Pa})$

$\varphi=$ flow coefficient

$\psi=$ total pressure coefficient 
$[\mathrm{K}]=$ FEM stiffness matrices

$[\mathrm{C}]=$ FEM damping matrices

$[\mathrm{M}]=$ FEM mass matrices

$\mathrm{F}(\mathrm{t})=$ external force

MPFs $=$ modal participation factors

$\boldsymbol{\Phi}=$ mode shape of the structure

$\mathrm{Y}=$ mode coordinate of the structure

$\omega_{n}=$ natural frequency

$\zeta=$ damping ratio

$\lambda=$ frequency ratio

$\operatorname{Re}=$ real part

$v_{n}^{*}=$ the node velocity of volute surface

$W_{\text {o,active }}=$ active output power

$\mathrm{x}(\mathrm{t})=$ vibrational displacement

$\dot{x}(t)=$ vibrational velocity

$\beta_{i}=$ amplification factor

$\rho=$ density, $\rho=1.20 \mathrm{~kg} / \mathrm{m}^{3}$

$\omega=$ angular velocity $(\operatorname{rad~s}-1)$

$\mathrm{FEM}=$ finite element method

$\mathrm{AML}=$ automatically matched layer

$a_{f e i}=$ active vibration acceleration value of each frequency in the spectrum

$a_{\text {ref }}=1 \times 10^{-6}(\mathrm{~m} \mathrm{~s}-2)$

$\mathrm{P}_{\mathrm{i}}=$ the source node pressure load $(\mathrm{i}=1,2,3,4)$

$\mathrm{P}_{\mathrm{A}}=$ the target node pressure load

$\mathrm{d}_{\mathrm{i}}=$ the distance from the source node to the target node $(\mathrm{i}=1,2,3,4)$

$N_{e}=$ the number of finite elements on the structural casing;

$S_{j}=$ the area of $\mathrm{jth}$ finite element, $\mathrm{m}^{2}$;

$P_{j}=$ the sound pressure of $\mathrm{jth}$ finite element, $p_{a}$;

$v_{n j}^{*}=$ the normal velocity of $\mathrm{jth}$ finite element, $m \cdot s^{-1}$.

$W_{S}=$ vibrational sound power

$M_{T}=$ total mass of volute

$S_{F T}, S_{S T}, S_{B T}=$ area of FT, ST and BT respectively

$I=$ structural surface acoustic Intensity

$\mathrm{DOE}=$ design of experimental

$\mathrm{RBF}=$ radial basis functions

$\mathrm{SPLs}=$ sound pressure levels $(\mathrm{dB})$

\section{Description of experimental procedures}

\subsection{Test fan description}

The test machine was a ventilating centrifugal fan with four main components (conical bell mouth, shrouded impeller, volute casing with conventional tongue and conical flow rates throttle) driven by an AC inverter motor with adjustable angular speed between $0 \sim 3600 \mathrm{r} / \mathrm{min}$; the design rotational speed was specified as $2920 \mathrm{r} / \mathrm{min}$, just as shown in Figure 1 . The main dimensions and characteristics of the investigated fan for this study are presented in Table 1. The ambient air was intake from the inlet pipe. The tests for characterizing the aerodynamic and acoustic behaviors of the fan system were made in a resilient installation to fulfill ship system noise and vibration requirements (according to standards GJB4058-2000 China [21] and GB-T1236-2000 China [22].) Figure $2 \mathrm{a}$ shows the details of the test system installation and data collection procedure. The following maximum measurement errors were obtained for the different magnitudes: total pressure $\pm 2 \%$ ( $\pm 10 \mathrm{~Pa})$, flow rate: $\pm 2 \%$ ( $\pm 0.05 \mathrm{~m} 3 / \mathrm{s})$ and shaft power $\pm 2 \%$ $( \pm 50 \mathrm{~W})$. 


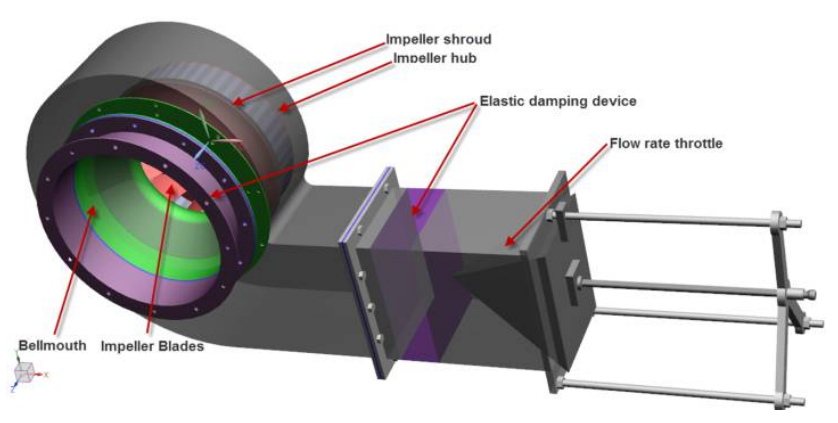

Figure 1 Component representation for the test fan

Table 1 Fan dimensions

\begin{tabular}{ll}
\hline Impeller blade outlet diameter $\mathrm{D}_{2}(\mathrm{~mm})$ & 520 \\
Impeller blade inlet diameter $\mathrm{D}_{1}(\mathrm{~mm})$ & 328 \\
Impeller outlet width $\mathrm{b}_{2}(\mathrm{~mm})$ & 102 \\
Impeller inlet width $\mathrm{b}_{1}(\mathrm{~mm})$ & 138 \\
Blade number $\mathrm{Z}_{\mathrm{n}}$ & 12 \\
Volute width $\mathrm{B}(\mathrm{mm})$ & 286 \\
Impeller-tongue distance $(\mathrm{mm})$ & 38 \\
Impeller-tongue distance $\left(\%\right.$ of $\left.\mathrm{D}_{2}\right)$ & $13.2 \%$ \\
\hline
\end{tabular}

\subsection{Dynamic pressure measurement}

In this part The Dynamic Pressure Testing System (DPTS) was employed to obtain the information of the casing pressure fluctuations. This system contained XCQ-080-5G Kulite high frequency dynamic pressure sensors, standard power supplies, 8300 AU amplifiers and AVANT MI-7016 signal acquisition. The test system installation is shown in Fig. 2b. There are 44 measurement locations on the casing surface, which were at five locations (p01-p05) in the vicinity of the volute tongue, and another six monitoring locations (p06-p11) were evenly distributed along the circumferential direction of the volute. Table 2 summarizes the angular coordinates of the measurement positions over the volute surface. The locations of monitoring points are shown in Fig. 3. The origin of the angle is the volute tongue. At each angular position, four axial measurements were made at the following $\mathrm{Z} / \mathrm{B}$ coordinates: $0.17,0.27,0.34$ and 0.75 (B is the volute axial width, and $\mathrm{Z}$ is the axial measurement position from the volute rear casing). $\mathrm{Z} / \mathrm{B}=0.07$ corresponds to the impeller hub, and $\mathrm{Z} / \mathrm{B}=0.36$ corresponds to the impeller shroud. More details about the DPTS installation and test have been reported in the previous work (Jianhua Zhang et al.) [23]

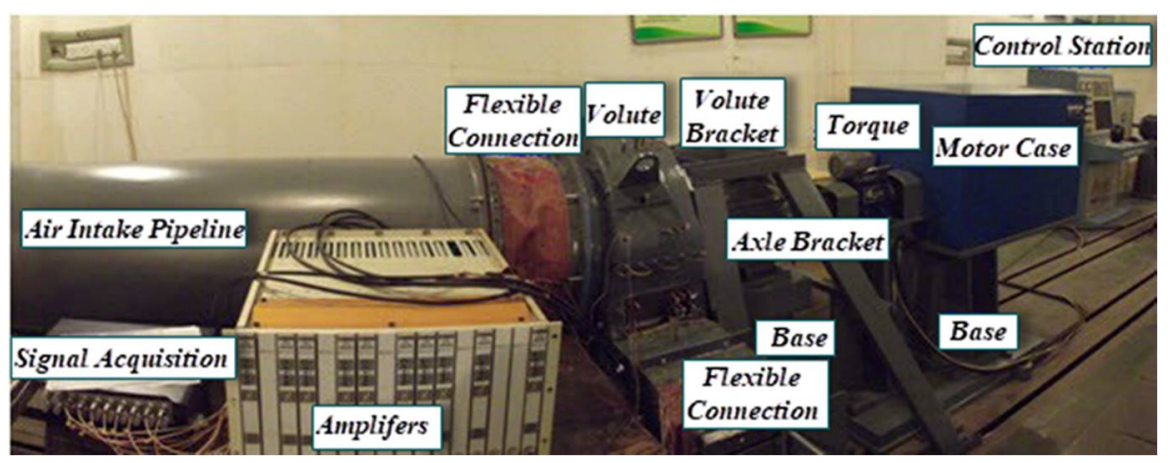

(a)

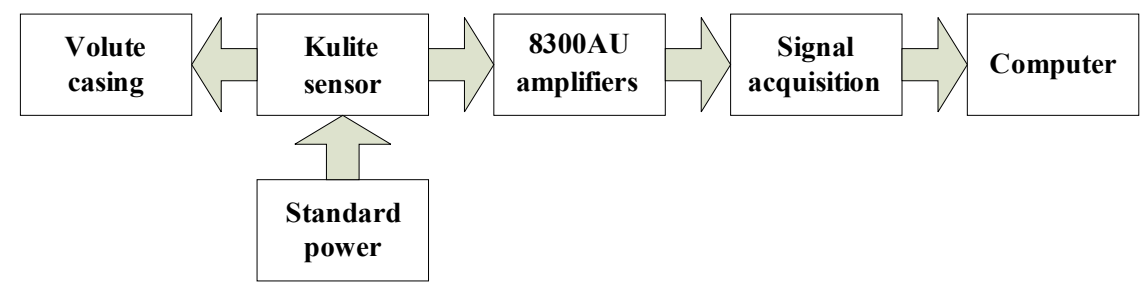

(b)

Figure 2 Installation of test system 

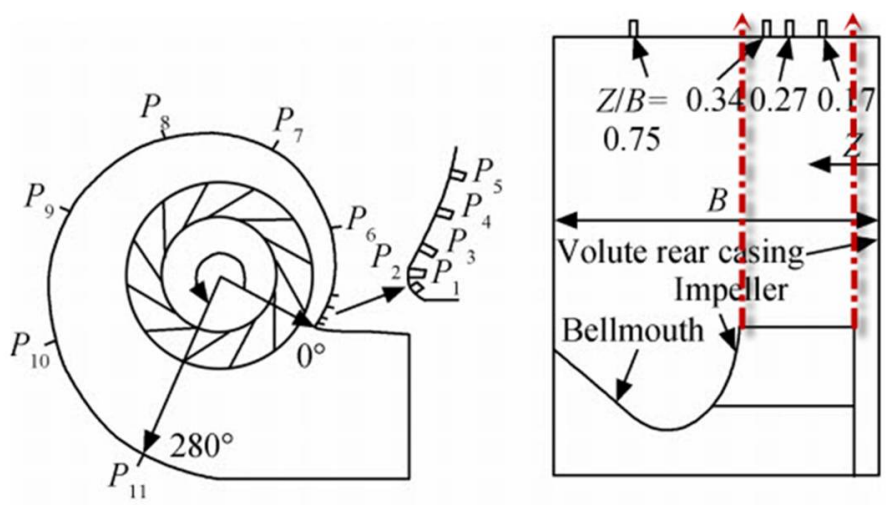

Figure 3 Sketches of the fan with the measurement Points

Table 2 Angular coordinate of the measurement points over the volute

\begin{tabular}{cccc}
\hline $\begin{array}{c}\text { Tongue } \\
\text { points }\end{array}$ & $\begin{array}{c}\text { Angular } \\
\text { Position }^{\left({ }^{*}\right.} \text { ) }\end{array}$ & $\begin{array}{c}\text { Volute } \\
\text { points }\end{array}$ & $\begin{array}{c}\text { Angular } \\
\text { Position( }\end{array}$ \\
\hline P1
\end{tabular}

2.3 Volute vibration test measurement

The LMS Test Lab test system was used to complete the vibrational test of the fan casing. To eliminate vibrational disturbances on the volute originating from the imported pipe and outlet throttle flow, elastic connections were used in two positions: at the connection between the transition section of an inlet and the volute, and between the volute outlet and the throttle valve. The flexible installation should meet the requirements of GJB4058-2000 (The Noise and Vibration Measurement Method of Ship Equipment) [21]. There is some major equipment required for this test, such as an LMS SC310W signal analyzer, a B\&K 4513 accelerometer, and a B\&K 4514 accelerometer. The background noise is ignored because of the lower value compared to the actual value of the fan. One hand of the accelerometer is fixed on the volute by bonding, and the other hand is directly connected to the data processing and analysis notebook by the data line. The arrangement of the vibration sensor is shown in Fig. 4 . There are 16 vibration measurement locations on the casing surface. The No. 1 to 5 \# measuring points are arranged near the border between the back panel of the volute and the side panel of the volute. The No. 1 measuring point is located near the tongue, and the other measuring points No. 2 to 5 \# are respectively arranged at the positions of 0 degrees, 90 degrees, 180 degrees, and 270 degrees. The No. 6 to 9 \# measuring points that are located at the edge of the plate between the back panel of the volute and the motor along with the connecting plate in the circumferential direction are arranged with an interval of 90 degrees. At the front panel of the volute with a 90-degree interval in the direction of a counterclockwise rotation layout are the No. 10 to 12 \# measuring points. The vibration measuring point of the volute side panel is in the middle of the axial width of the volute arranging the No. 13 to 15 \# measuring points. The No. 13 \# measuring point is defined as the starting point; the No. 14 \# and 15 \# measuring points are also arranged in the side volute at an interval of 140 degrees; and the No. 16 \# measuring point is at the outlet of the volute side panel. The vibration test and the dynamic pressure test of the volute are carried out at the same time, and the data are, respectively, collected in different control computers. Then, the data are sequentially extracted to complete the post-processing.
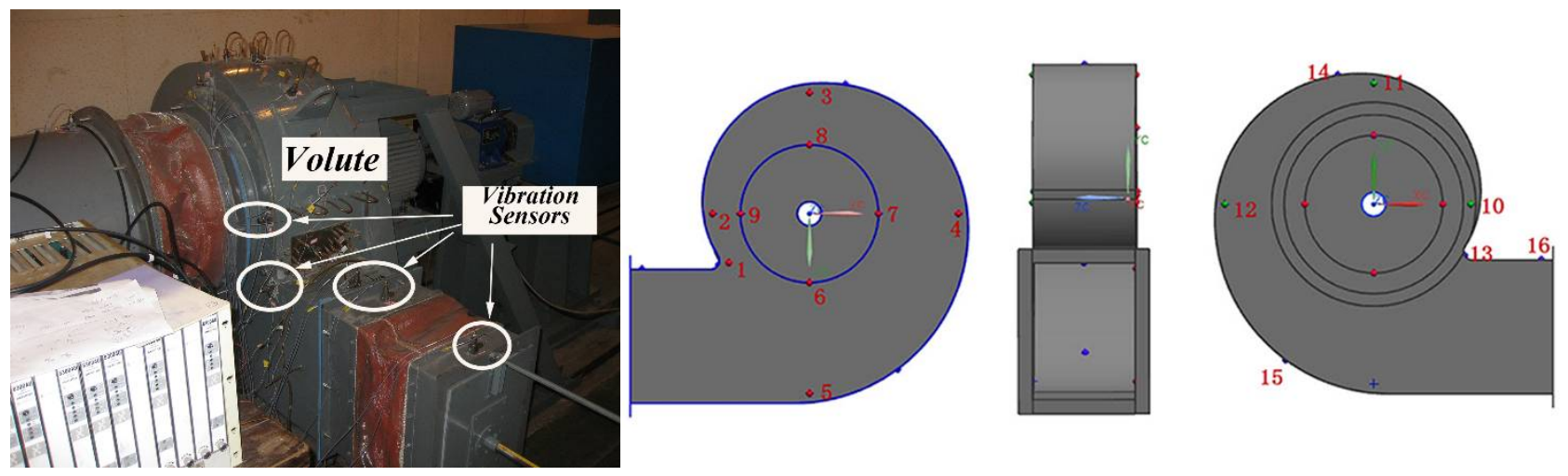
(a) the distribution of measuring points of experimental

(b) the distribution of measuring points of simulation

Figure 4 The arrangement of the vibration sensor

\section{Unsteady flow simulation and validation}

\subsection{Numerical methods}

In this paper the complete unsteady flow for the entire impeller-volute configuration was conducted using the computational fluid dynamics (CFD) code ANSYS CFX. The code numerical simulation is based on a finite-volume numerical method that employs an incompressible turbulent flow model to solve the continuity equation and the three-dimensional time-averaged NavierStokes equations. The characteristic Mach number of the simulated fan described by the blade tip circumferential velocity was $\mathrm{u}_{2} / \mathrm{c}=0.18(<0.3)$, and the flow was therefore guaranteed to be incompressible. The continuity equation and momentum equations were solved independently of the energy equation because of the isothermal flow. The Standard $k-\varepsilon$ turbulence model, which was applied to capture wall pressure fluctuations by Ballesteros-Tajadura [24] and Cai [25], was used in the present simulation of the unsteady flow field. A coupled solver, which uses a fully implicit discretization scheme to solve all of the equations (corresponding to the velocity and pressure), was used. However, a second order high-resolution discretization scheme was used for the convection terms, and a second order backward Euler scheme was used for the transient terms.

For the three-dimensional calculations, a couple of high quality hexahedral structural grids were employed to define the flow domains. Details of the grid features and meridian grid cross-section, including the radical gap and cavity around the volute, are shown in Figs. 5. More details about the grids of this fan have been reported in the previous work (Jianhua Zhang et al.)[23] Numerical deviations strong connections with the grid number needed to be removed, and a grid-independent validation of the fan total pressure coefficient and efficiency was performed. Figure 6a shows the influence of the grid size on the fan total pressure coefficient and efficiency. From the figure, we can see that the grid was independent when the total number of grid points exceeded 2.8 million. In addition, by increasing the grid size to 5.7 million, the total pressure coefficient with respect to the flow rate was nearly unchanged, compared with the smaller total pressure coefficient over a small flow rate range close to the best efficiency point (BEP).

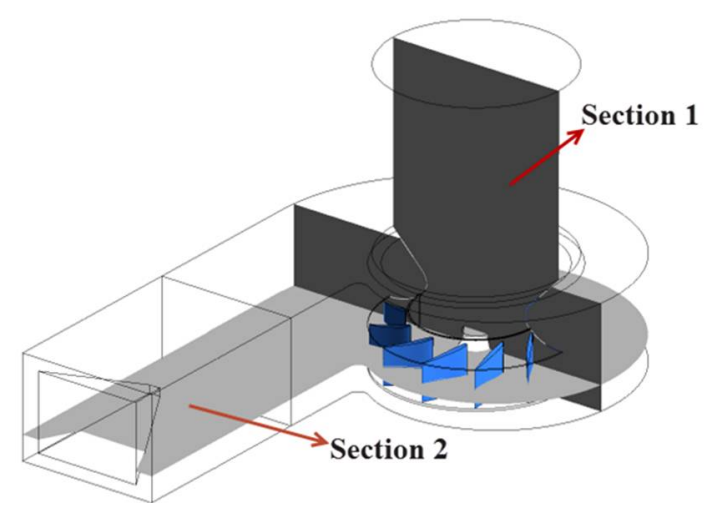

(a) Section workspaces

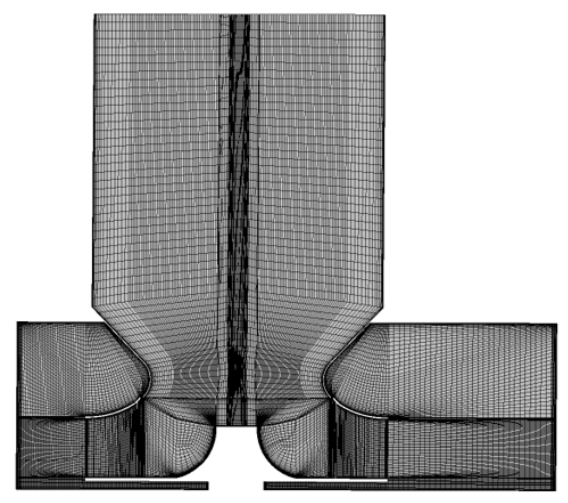

(b) Mesh details of section 1

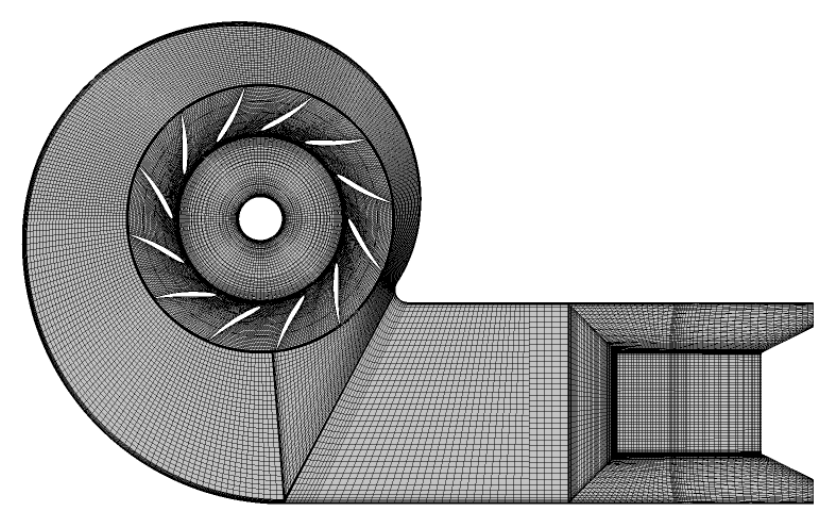

(c) Mesh details of section 2

Figure 5 Mesh details of fan

The modeled boundary conditions were considered to have greater physical meaning for turbomachinery flow simulations. In this case, the CFD simulation process began with a steady flow calculation using the frozen-rotor approach, and nonslip conditions 
were specified at the solid walls. In addition, for the near-wall flow region, a scalable wall function treatment based on the logarithmic law [26] was applied to cause the mean value of Yplus to vary between 30 and 300, as shown in Fig. 7. Therefore, by applying this method, the number of the grid points was greatly reduced without reducing the accuracy of the calculations. For the unsteady flow, a transient rotor/stator grid interface based on sliding grid technique was applied, which allowed unsteady interactions between the impeller and volute casing. A time step of $5.7089 \mathrm{e}^{-5} \mathrm{~s}$ was used for the calculation of the unsteady interactions, which was sufficient for the dynamic analysis. The time step was related to the rotational speed of the impeller, and the time step was specified such that the impeller rotated once in 360 steps (a blade passage defined 30 steps). The number of iterations was adjusted to reduce the residual below an acceptable value at each time step. At each time step, a reduction of $10^{-5}$ (five orders of magnitude) in the residuals for the given variables in the cells was required. The unsteady simulation was initialized using a steady solution, and over 15 revolutions (approximately 5400 time steps) were required to converge on a periodic unsteady solution.

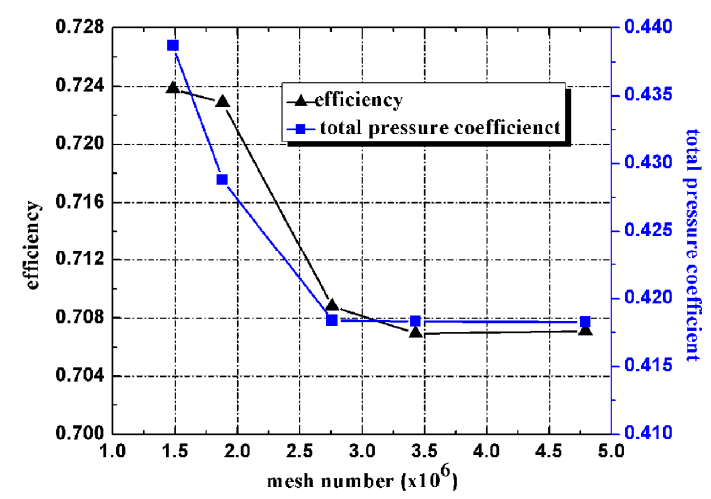

(a)

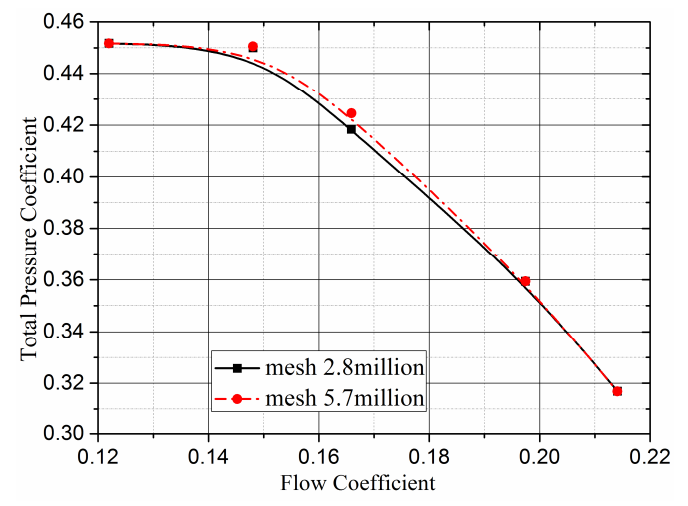

(b)

Figure 6 Grids size independent: (a) fan total pressure and efficiency with grids size; (b) total pressure coefficient with flow rate

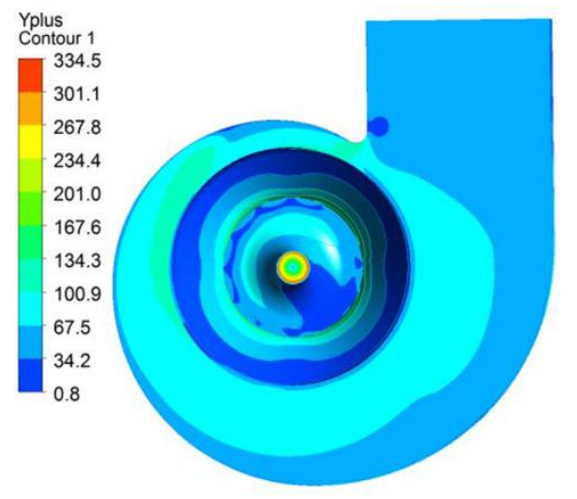

Figure 7 Yplus of the simulated fan

3.2 CFD results validation

\subsubsection{Overall performance validation}

Using the defined test bench, the overall performance of this fan predicted by the CFD calculations was compared for different flow rates. The non-dimensional flow rate and the total pressure rise were transformed by

$$
\begin{aligned}
& \varphi=Q /\left(D_{2}^{2} u_{2} \pi / 4\right) \\
& \psi=p_{t} / \rho u_{2}^{2} .
\end{aligned}
$$

The best efficiency point (BEP) at a rotational speed $2920 \mathrm{rpm}$ corresponded to a flow rate $\mathrm{Q}=3.3614 \mathrm{~kg} / \mathrm{s}(\varphi=0.1659)$ and a total pressure rise $\mathrm{P}_{\mathrm{T}}=3182 \mathrm{~Pa}(\psi=0.41954)$. Figure 8 indicates that the measured total pressure coefficient and efficiency agreed well with the three-dimensional steady-state calculations. The expected trend of decreasing total pressure coefficients with increase flow rates can be observed in the experimental and numerical curves. In addition, the performance curves between numerical simulation and experimental test results were perfectly consistent for larger grids (nearly double the size of the small grids). 


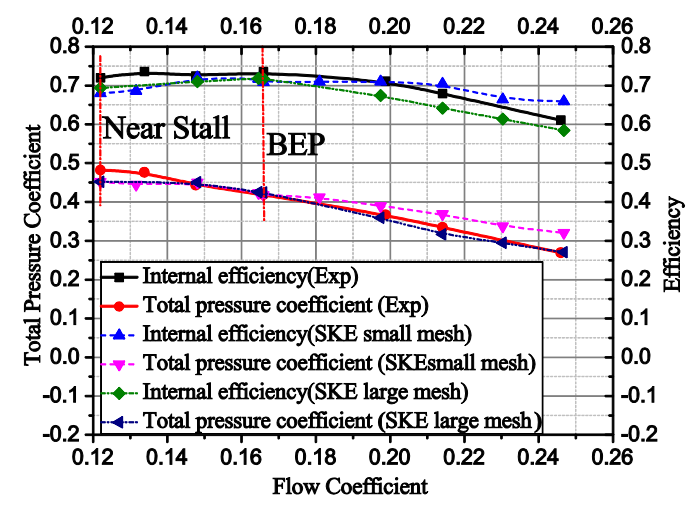

Figure 8 Comparison between numerical and experimental curves

\subsubsection{Wall pressure fluctuation validation}

The unsteady aerodynamic forces on the volute casing caused by the unsteady flow of the impeller outlet are the significant vibrational sources; thus, the validation of the numerical results of the unsteady aerodynamic forces are very important. In this study, to confirm the simulation accuracy, a comparison analysis between the dynamic pressure measurement results of volute casing and the numerical calculation was conducted. Because of the similar spectrum signals of the different measurement points on the volute surface, only a few typical points were selected for analysis and confirmation. Figure 9 shows the power spectra of the volute pressure fluctuations at the BEP at the typical selected measurement point 01, point 06 and point 09 . The left figure shows the experimental measurement results, and the right figure shows the numerical results. It was something of a satisfaction to find that the numerical and experimental amplitude of the BPF which presented the predominant frequency component were in good agreement at three most important axial positions $(\mathrm{Z} / \mathrm{B}=0.17,0.27,0.34)$. More details about the results discussion have been reported in the previous work (Jianhua Zhang et al.)[23]
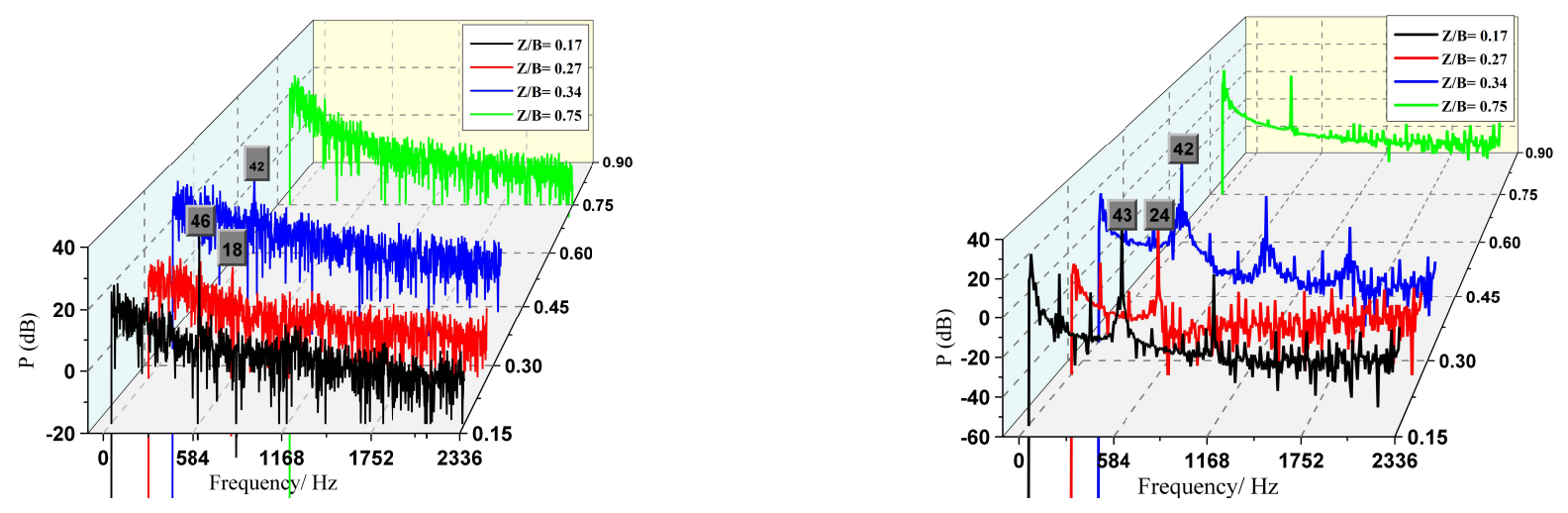

(a)
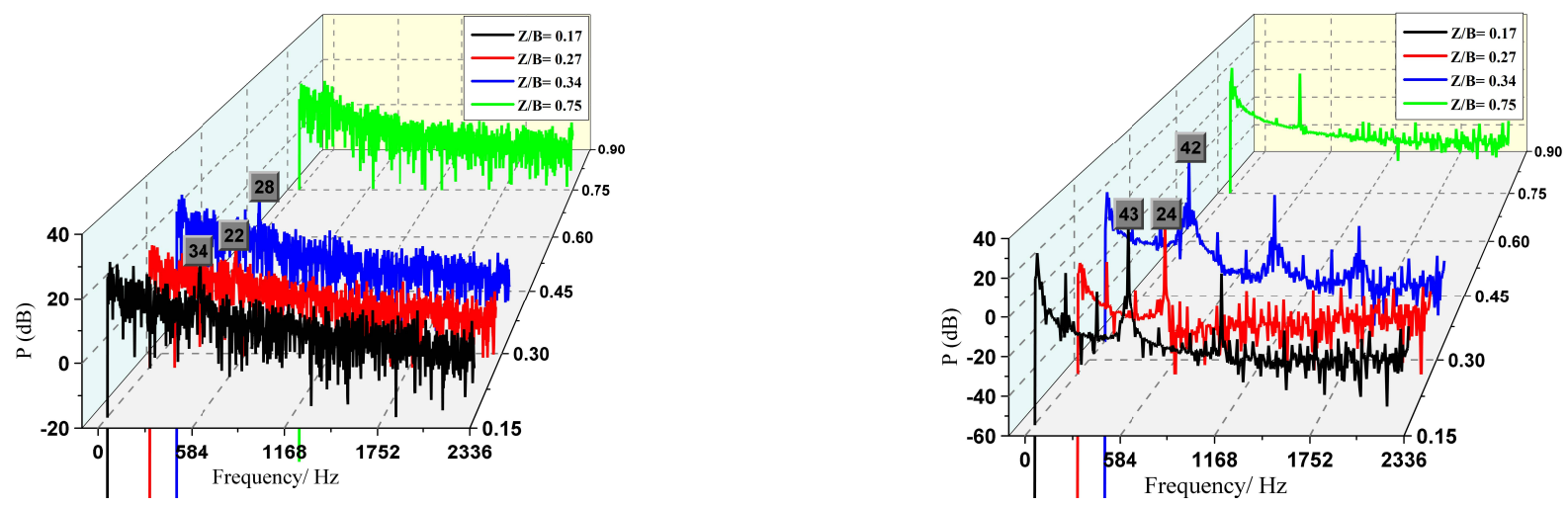

(b) 

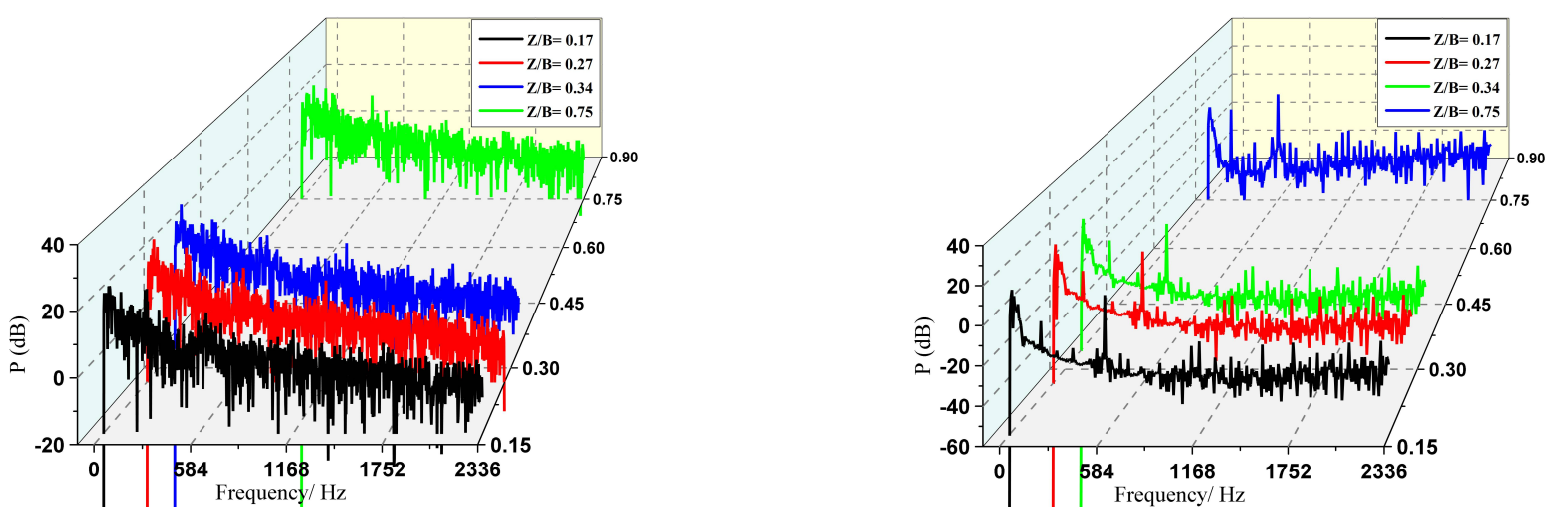

(c)

Figure 9 Power spectra of volute pressure fluctuation at three measurement points with the flow rate BEP Point 01 (exactly at the tongue); Point 06 (52 ${ }^{\circ}$ from the tongue) and Point 09 (187 degree from the tongue)

\section{Volute vibro-acoustic model, FEM Validation and Sound Estimation}

The studied marine centrifugal fan was operating in a completely enclosed small place, and the fan inlet and outlet were connected with a long pipe; thus, the aerodynamic noise caused by the fan internal flow during operation cannot be directly spread to the outside. Only the vibrational noise can spread to the outside through the vibrations occurring as part of the volute casing vibrations. With the fan running, the internal unsteady flow motivated the casing vibrations, and the volute surface was slightly deformed. However, the displacement of the volute surface deformation is much smaller than the boundary layer thickness of the fluid wall. Therefore, one-way fluid-solid coupling was applied in the simulation. Jiang et al. [27] applied a unidirectional coupling technique that validated the rationality of an unsteady flow-induced vibration of a centrifugal pump. The rationality of unidirectional coupling is also verified in this study. For details please refer to section 4.3.

4.1 Vibro-acoustic mathematical model

For a continuous system of an actual structure, dispersed by FEM, the dynamic balance equation is as follows:

$$
\mathbf{M} \ddot{x}(t)+\mathbf{C} \dot{x}(t)+\mathbf{k} x(t)=\boldsymbol{F}(t)
$$

As the structure is subjected to external harmonic force, the external force can be expressed as follows:

$$
\boldsymbol{F}(t)=\boldsymbol{F} \cdot e^{j w t}
$$

The modal vectors are linearly independent of each other. Therefore, the response of the dynamic under any excitation can be regarded as the coupling of the systematic modes and the modal participation factors (MPFs) of each order. At this point, the displacement response can be expressed as follows:

$$
\boldsymbol{x}(t)=\sum_{i=1}^{n} \boldsymbol{\varphi}_{i} y_{i}(t)=\mathbf{\Phi} \mathbf{Y}
$$

In the formula, $\varphi_{i}$ represents the ith mode shape of the structure, $y_{i}(t)$ represents the ith mode coordinate, which is called the ith MPFs; $\boldsymbol{\Phi}=\left[\boldsymbol{\varphi}_{1} \boldsymbol{\varphi}_{2} \cdots \boldsymbol{\varphi}_{n}\right] ; \quad \mathbf{Y}=\left[y_{1} y_{2} \cdots y_{n}\right]$.Substituting (5) into (3) and multiplying $\boldsymbol{\Phi}^{T}$ on the two lateral yields the following:

$$
\boldsymbol{\Phi}^{T} \mathbf{M} \ddot{\mathbf{Y}}+\boldsymbol{\Phi}^{T} \mathbf{C} \boldsymbol{\Phi} \dot{\mathbf{Y}}+\boldsymbol{\Phi}^{T} \mathbf{K} \boldsymbol{\Phi} \mathbf{Y}=\boldsymbol{\Phi}^{T} \boldsymbol{F} \mathrm{e}^{j \omega t}
$$

Using the orthogonality of modal vectors for the mass, damping, and stiffness matrixes, we obtain independent coefficients of the single degrees of freedom of $\mathrm{n}$ items. Therefore, the original system can be regarded as linear superposition independent coefficients of single degrees of freedom of $\mathrm{n}$ items.

$$
\text { Ordering, } \boldsymbol{\Phi}^{T} \mathbf{M} \boldsymbol{\Phi}=m_{i}, \quad \boldsymbol{\Phi}^{T} \mathbf{C} \boldsymbol{\Phi}=C_{i}, \quad \boldsymbol{\Phi}^{T} \mathbf{K} \boldsymbol{\Phi}=K_{i}, \quad \boldsymbol{\Phi}^{T} \boldsymbol{F}=\boldsymbol{F}_{i}
$$

Substituting (7) into (6), the transformation is as follows: 
$m_{i} \ddot{y}_{i}(t)+C_{i} \dot{y}_{i}(t)+K_{i} y_{i}(t)=F_{i} e^{j \omega t}$

Ordering, $\omega_{n}=\sqrt{k_{i} / m_{i}}, \quad \zeta_{i}=c_{i} / 2 \sqrt{k_{i} m_{i}}$

Substituting (9) into (8) results in the following:

$\ddot{y}_{i}(t)+2 \zeta_{i} \omega_{n} \dot{y}_{i}(t)+\omega_{n}^{2} y_{i}(t)=F_{i} e^{j \omega t}$

Using the theory of ordinary differential equations, we obtain the stable solution of (4) as follows:

$y_{i}(t)=\frac{F_{i}}{\omega_{n}^{2}-\omega^{2}+2 j \zeta_{i} \omega \omega_{n}} e^{j \omega t}$

Introducing the frequency ratio $\lambda=\omega_{n} / \omega$ and the dimensionless vibration mode amplification factor $\beta_{i}$ results in the following:

$\beta_{i}=\frac{1}{\sqrt{\left(\lambda^{2}-1\right)^{2}+\left(2 \zeta_{i} \lambda\right)^{2}}}$

Ordering, $\psi_{i}=\arctan \frac{2 \zeta_{i} \lambda}{\lambda^{2}-1}$

Substituting (12) and (13) into (11) results in the following:

$$
y_{i}(t)=\frac{F_{i}}{\omega^{2}} \beta_{i} e^{j(\omega t-\psi)}
$$

At this point, the vibrational displacement is as follows:

$$
\boldsymbol{x}(t)=\sum_{i=1}^{n} \boldsymbol{\varphi}_{i} y_{i}(t)=\sum_{i=1}^{n} \boldsymbol{\varphi}_{i} \frac{F_{i}}{\omega^{2}} \beta_{i} e^{j(\omega t-\psi)}
$$

The vibrational velocity is as follows:

$\dot{\boldsymbol{x}}(t)=\sum_{i=1}^{n} \boldsymbol{\varphi}_{i} \dot{y}_{i}(t)=\sum_{i=1}^{n} \boldsymbol{\varphi}_{i} \frac{F_{i}}{\omega} \beta_{i} e^{j\left(\omega t-\psi+\frac{\pi}{2}\right)}$

The active output power is as follows:

$W_{o, \text { active }}=\int_{S} \operatorname{Re}\left(I_{n}\right) d s=\frac{1}{2} \int_{S} \operatorname{Re}\left(p v_{n}^{*}\right) d s$

The relationship between the plane wave sound pressure $\mathrm{p}$ and the surface velocity $v_{n}^{*}$ is as follows:

$p=\rho_{0} c_{0} v_{n}^{*}$

Substituting (18) into (17) results in the following:

$W_{o, \text { active }}=\int_{S} \operatorname{Re}\left(I_{n}\right) d s=\frac{1}{2} \rho_{0} c_{0} \int_{S} \operatorname{Re}\left(v_{n}^{* 2}\right) d s$

Substituting (16) into (19) results in the following:

$$
W_{o, a c t i v e}=\int_{S} \operatorname{Re}\left(I_{n}\right) d s=\frac{1}{2} \rho_{0} c_{0} \int_{S} \operatorname{Re}\left(\sum_{i=1}^{n} \boldsymbol{\varphi}_{i} \frac{F_{i}}{\omega} \beta_{i} e^{j\left(\omega t-\psi+\frac{\pi}{2}\right)}\right)^{2} d s
$$

According to Eq. (30), it can be concluded that the structural acoustic radiation power is mainly determined by the modal shape $\varphi_{i}$, the applied exciting force $F_{i}$, and the frequency amplification factor $\beta_{i}$. Therefore, the following methods can be used to control vibrational noise: 
(1) With the structural model and material determined, the vibrational sound radiation can be weakened by attenuating the amplitude of the applied exciting force;

(2) With the determination of exciting force, the geometric parameters of the structural model are modified to reduce the modal shape;

(3) To reduce the amplitude amplification factor, the frequency of the exciting force should avoid the natural frequency.

Regarding the studied fan volute structure, the structural mode can be changed by controlling the thickness distribution of the structure, if the geometry, the stiffness and constraint position are fixed.

\subsection{Volute FEM model and Validation}

\subsubsection{Structural FEM model}

In this study, NX Nastran, the commercial software made by Siemens Company, was used to calculate the modal and vibration response of the volute. The finite element model (FEM) of the volute was selected by using a high-quality surface quadrilateral mesh, as shown in Fig.10. The thickness of the volute panel is relatively small (up to $6 \mathrm{~mm}$ ), and the shell63 element is selected for the FEM, as the shell63 element has both the bending and membrane capabilities, and can suffer both plane and normal loads. The volute FEM with a total of 46,182 shell63 element grids was divided into three main sections according to the different thickness properties. The front panel thickness (FT) and the back panel thickness (BF) were set to $6 \mathrm{~mm}$, and the volute side panel thickness was set to $5 \mathrm{~mm}$ (ST). In addition, the model material was steel, the density $\rho=7800 \mathrm{~kg} / \mathrm{m}^{\wedge} 3$, the elastic modulus $=2.06 \mathrm{e} 11 \mathrm{pa}$, and Poisson's ratio $v=0.3$. The volute casing was fixed to a supporting stand by ten fastening bolts at the casing front. The volute panel rear (near the motor) was connected by four fixed bolts, and the three translational degrees of freedom of the nodes at the bolts were restricted to zero. The panel thickness distribution and the degree of freedom constraints on the volutes are shown in Fig. 10.

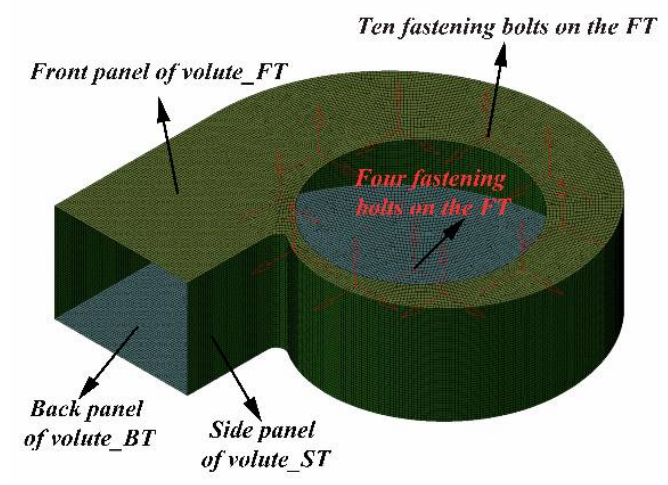

Figure 10 The finite element model (FEM) of the volute

\subsubsection{Acoustic FEM model}

The volute acoustical FEM model is shown in Fig. 11. It was similar to the acoustical finite element mesh used for aerodynamic noise calculations [23]. Taking into account the characteristic of radiated vibrational noise, the volute's inlet and outlet were completely enclosed. More importantly, according to the requirements on element size driven by maximum frequency, the computational acoustic mesh had to satisfy each wavelength corresponding to six elements. An acoustical mesh with a maximum element size of $15 \mathrm{~mm}$ was applied in the sound computation and guaranteed a spatial resolution at the maximum frequency of 3236 $\mathrm{Hz}$ of six points per wavelength. Atmospheric boundary layer (AML) boundary layers were introduced to simulate the unbound boundary of the exterior fluid domain. The outermost layer exposed to the AML surface that satisfied the Sommerfeld radiation condition was defined as a non-reflecting boundary. Then, a field point mesh based on standard ISO3744 [28] that enclosed the entire calculation domain was established using an approximate free-field engineering method. 


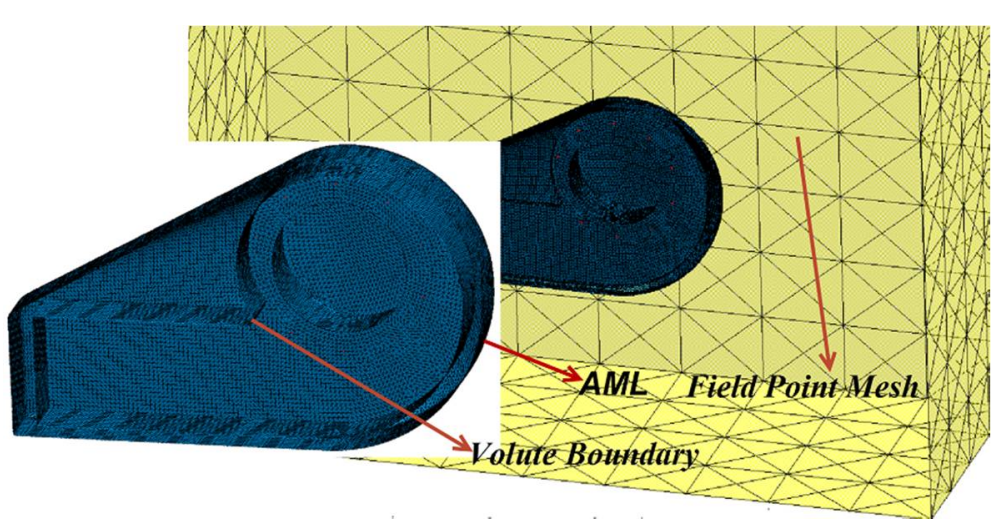

Figure 11 The volute acoustical FEM model

\subsection{Volute vibration validation}

In order to verify the rationality of the unidirectional fluid-structure interaction method, the vibration measurement of the volute casing induced by unsteady flow is carried out in detail, based on the vibrational measuring points, and arranged as shown in Fig.4. Most importantly, it should be stated that the volute casing vibration measurements, the vibration response calculation and the vibrational noise production are all carried out under the fan design flow point, the best-efficiency point (BEP).

The definition of the total vibrational amplitude is as follows [29]:

$$
V A L=\sqrt{\sum_{i=1}^{n} a_{f e i}^{2}}
$$

Where, $a_{f e i}$ represents the single frequency valid values of vibration acceleration in the spectrum.

The calculated vibrational measurement positions are arranged according to the vibrational test. Fig. 12 shows a comparison of the numerical and experimental results of the total vibration levels of various vibrational positions in the range of 20 to $3000 \mathrm{~Hz}$. As seen in the figure, the calculations are in good agreement with the experiments, the detailed results and the analysis of [29]. Moreover, a comparison between the experimental and the numerical results shows that it is reasonable and effective to adopt the one-way fluid-structure-acoustic coupling method. Figure 13 presents the vibration acceleration spectrum of the selected three measuring positions (corresponding to the volute rear panel [BT], the volute front panel [FT], and the volute side panel [ST]). It can be seen from the figure that the spectrum waveforms at each measuring position are similar, and the maximum amplitude of vibration acceleration presents at the fundamental frequency, indicating that the fundamental frequency, the blade passing frequency (BPF), is the major component for volute vibrations induced by unsteady flow.

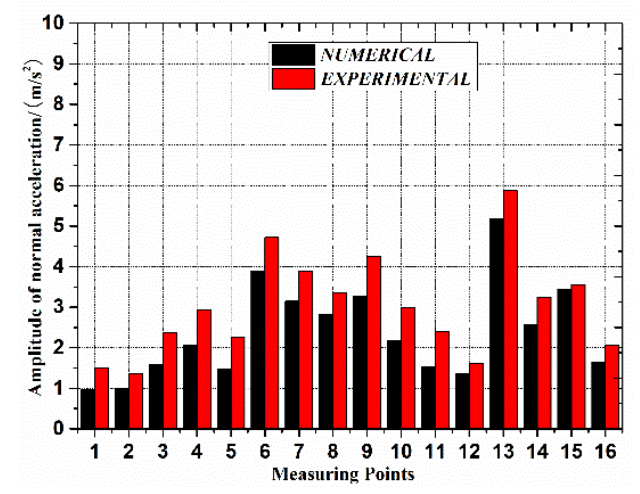

Fig. 12 The compassion of numerical and experimental amplitude of normal acceleration 


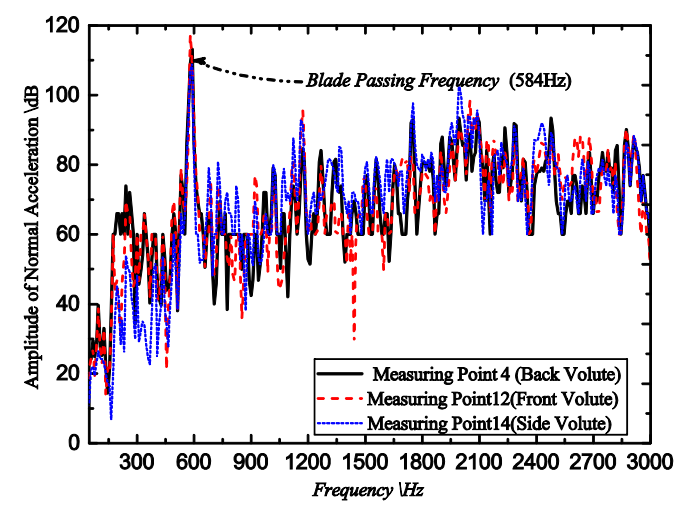

Fig. 13 The spectrum of acceleration on the three different measuring points

4.4 Volute vibro-acoustic Estimation

Figure 14 shows the numerical evaluation method of the volute vibro-acoustic coupling. It can be seen that the fluid-structureacoustic unidirectional coupling method is divided into three main steps: the first is acquisition of the vibrational source of the volute based on the unsteady flow calculation on the centrifugal fan, then transformation of the extracted time-domain fluctuation data into frequency-domain data through FFT, providing basic data for the next vibration response and vibro-acoustic calculation; the second is interpolation of the frequency-domain node pressure of the fluid into the corresponding structural FEM nodes according to equation (5) (Where $P_{i}(i=1,2,3,4)$ is the source node pressure load, $P_{A}$ is the target node pressure load, and $d_{i}(i=1,2,3,4)$ is the distance from the source node to the target node; Fig. 15 is a sketch of the geometric interpolation algorithm) and assignment of the interpolated node pressure of the structure to the boundary loads of vibro-acoustics, then application of the structural FEM to obtain the modal participation factor of the volute; the third is loading of the modal participation factor and vibro-acoustic boundary loads obtained during the second step, to calculate the volute vibrational sound radiation using the modal superposition vibroacoustic method.

$$
P_{\mathrm{A}}=\frac{P_{1} \frac{1}{d_{1}}+P_{2} \frac{1}{d_{2}}+P_{3} \frac{1}{d_{3}}+P_{4} \frac{1}{d_{4}}}{\frac{1}{d_{1}}+\frac{1}{d_{2}}+\frac{1}{d_{3}}+\frac{1}{d_{4}}}
$$

\section{Fluid-Solid-Acoustic Coupling Procedure}

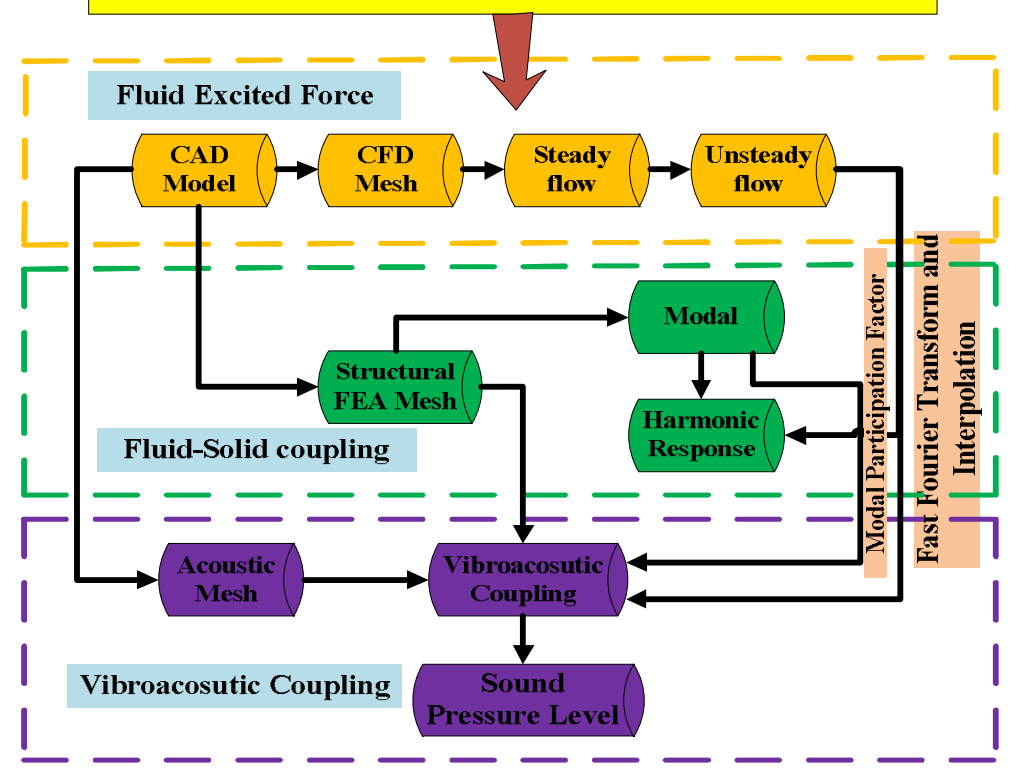

Figure 14 The flow chart of numerical evaluation method of volute vibro-acoustic coupling 


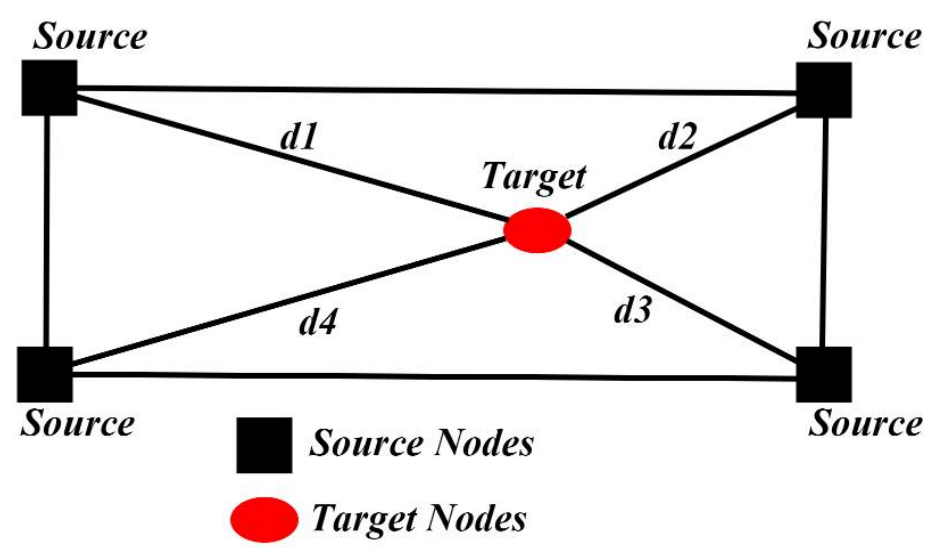

Figure 15 The diagram of the geometric interpolation algorithm

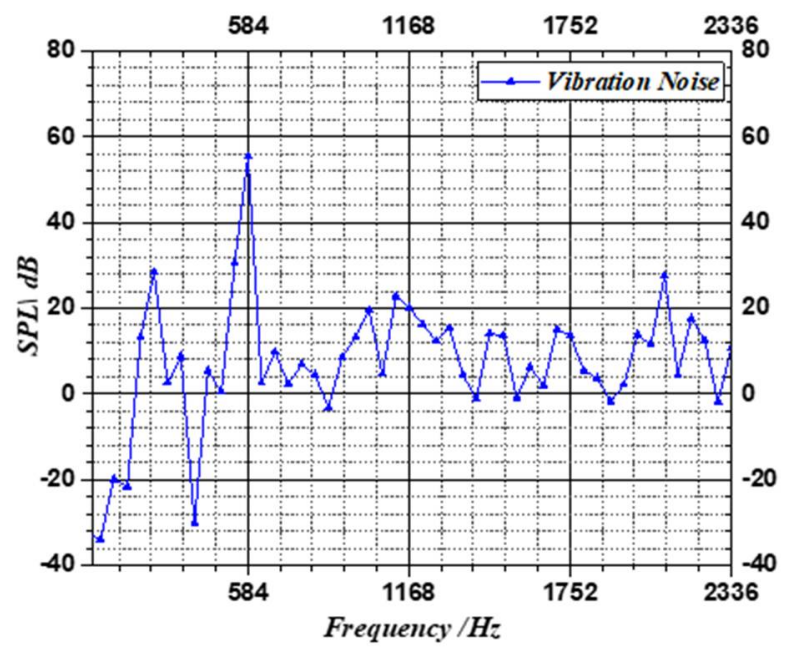

Figure 16 The spectrum program of vibrational sound radiation of the volute casing (Numerical)

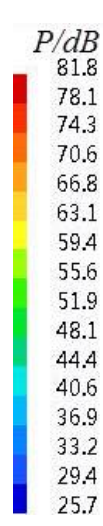

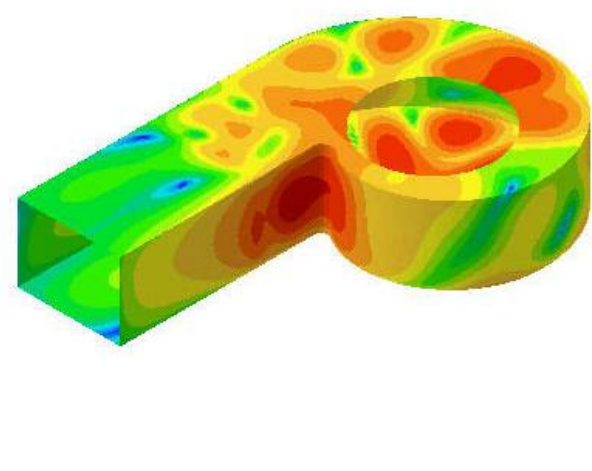

(a) Side

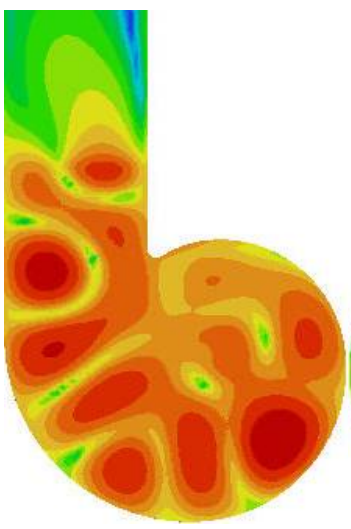

(b) Back

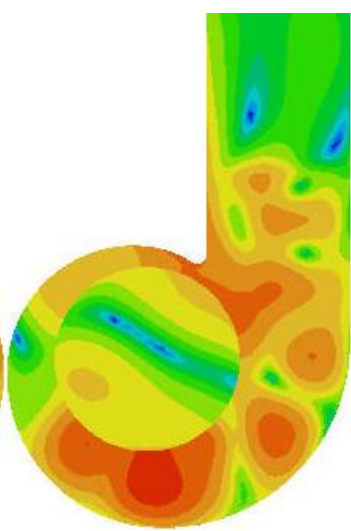

(c) Front

Figure 17 The distribution of the vibrational sound radiation of the volute casing surface at the fundamental frequency (BPF)

Fig. 16 presents the spectrum program of the vibrational sound radiation of volute casing, and the vibrational noise at the fundamental frequency (BPF) is obvious. Moreover, the vibrational sound radiation distribution of the volute casing surface at the fundamental frequency is presented in Fig. 17. It can be observed that the outlet of the volute side panel near the volute tongue region and the volute back panel at $180^{\circ}$ from the tongue presented very strong vibrational acoustic radiation values. In addition, the theoretical derivation of chapter 4.1 shows that the real part $\operatorname{Re}\left(p v_{n}^{*}\right)$ acoustic intensity of acoustical power characterized the vibrational acoustic energy, and it is determined by the product of the surface sound pressure $p$ and the surface normal conjugate velocity $v_{n}^{*}$, but is also a quadratic function of the vibrational velocity, Lu [16], indirectly reducing the volute surface acoustical radiation through a decrease in the surface normal velocity of the volute casing. 


\section{Vibro-acoustic Optimization}

\subsection{Optimization Objective Selection}

Section 4.1 of this paper provides two means to control vibro-acoustics: the control method (1) can be used to modify the geometrical shape of the volute tongue or wavy edge of the blade to weaken the pressure fluctuations on the volute casing, and control method (2) can be used to modify the model geometry, thickness distribution, and constrained position and reduce the modal shapes. However, the shapes and natural frequency are determined by the structural thickness distribution, structural stiffness, and the constrained position. The modal shapes can be changed by controlling the thickness distribution, when the geometry, stiffness, and constrained position of the structure are fixed.

Concerning the studied centrifugal fan volute with several welding panels, this study developed an optimization method of vibroacoustics by changing different panel thicknesses (FT, BT, and ST) to attenuate the volute vibrational acoustic radiation. Regarding vibro-acoustic optimization, we must first determine the optimization target function. From the literature review, it is generally considered that the uniform sound pressure at the received position or the radiated sound power at the structural surface should be set as the optimization function. The selection of the target function depends on the research problems, and the target function is divided into two categories according to different attributes. One is a noise optimization problem (internal noise control problem) applied in a closed domain. In this case, one or a few sound pressure levels of a specific measuring point is taken as the target function [17-20]. Concerning the noise control problem in the open domain, the external acoustic power on the structural surface was chosen as the optimal target function. This method has been proven by many scholars [17-18, 30].

For the type kind of target function, the sound pressure at the arranged receiving position can be clearly determined, but it may result in judgments of distortion, such as a low sound pressure value at an arranged position may be obtained and a high sound pressure value at other points can be presented. Thus, it is a very important to choose such target functions. Fortunately, Marburg [31] proposed an improved target function (F), which is defined as follows:

$$
F=\tilde{F}^{\frac{1}{n}}=\left(\frac{1}{\omega_{\max }-\omega_{\min }} \int_{\omega_{\min }}^{\omega_{\max }} \phi\left\{p_{l}(\omega)\right\} d \omega\right)^{\frac{1}{n}}
$$

In Eq. (23), $\varnothing\left\{p_{l}(\omega)\right\}$ represents the given weighting function, replaced by the following equation:

$$
\phi\left\{p_{l}(\omega)\right\}= \begin{cases}\left(p_{l}-p_{r e f}\right)^{n} & p_{l}>p_{\text {ref }} \\ 0 & p_{l}<p_{\text {ref }}\end{cases}
$$

$\mathrm{n}=1$, which is the average value of sound pressure in the frequency spectrum; and $\mathrm{n}=2$, which is the RMS value of sound pressure.

For the second type of problem, Koopmann and Fahnline [2] proposed an optimization method that takes the external radiated sound power as the target function, and it is suitable for the optimal noise control method in this study. They provided the root mean square (RMS) expression of sound pressure in an enclosed space as follows:

$$
W=\int_{S} \operatorname{Re}\left(I_{n}\right) d s=\frac{1}{2} \int_{S} \operatorname{Re}\left(p v_{n}^{*}\right) d s
$$

Where, S refers to the structural surface, and the structural surface is discretized based on FEM. At this time, the structural radiated sound power can be regarded as the sum of the individual radiated sound power on the FEM mesh [32]. Thus, after organization, Eq. (25) can be changed to the following:

$$
W=\sum_{\mathrm{j}=1}^{N_{e}} W_{j}=\frac{1}{2} \operatorname{Re}\left(\sum_{\mathrm{j}=1}^{N_{e}} \frac{1}{2} \int_{S_{j}} p_{j} v_{n j}^{*} d S\right)
$$

In the formula:

$N_{e}$ - the number of finite elements on the structural casing;

$S_{j}$ - the area of jth finite element, $\mathrm{m}^{2}$;

$P_{j}$ - the sound pressure of jth finite element, $p_{a}$;

$v_{n j}^{*}$ - the normal velocity of jth finite element, $m \cdot s^{-1}$. 
The vibrational noise of the volute structure of marine centrifugal fans studied in this paper belong to the typical external opening noise radiation problem. Therefore, the second type of optimization target function should be adopted.

\subsection{RBF approximation surrogate models}

\subsubsection{Design variables and parametric model}

The previous analysis shows that the structure modal shape can be changed by controlling the structural thickness distribution, so as to improve the structural vibration sound radiation. Therefore, the volute thickness of the three panels (FT, BT, and ST in mm) were specified as the design variables. Thus, the design objectives can be achieved by adjusting the combination of different volute panel thicknesses in the optimization design. The thickness of each volute panel is parameterized using FEM. Figure 18 shows the volute parameterization structural FEM. Because the panel thickness of each volute is generally less than $10 \mathrm{~mm}$, we assigned the panel thickness of each volute to be from $4 \mathrm{~mm}$ to $10 \mathrm{~mm}$.

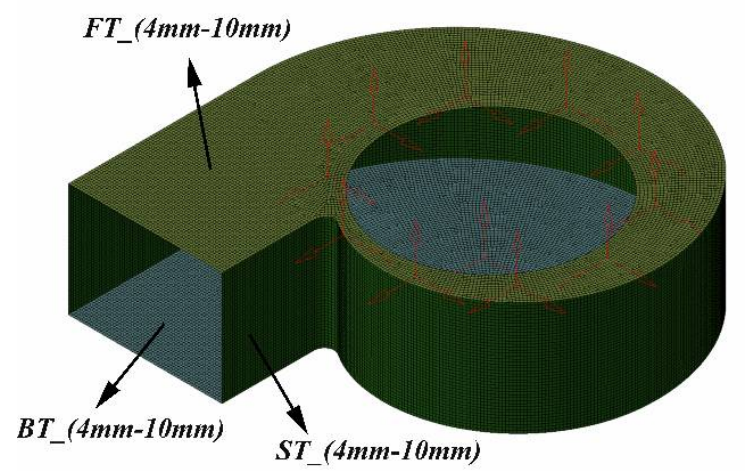

Figure 18 the volute parameterization structural FEM

\subsubsection{Design Space and RBF Approximation model Framework}

The optimization of the vibro-acoustics of the volute casing involves many combinations of different simulation programs, such as Unigraphics (UG) modeling, ANSA meshing, Nastran FEM analyzing, LMS acoustical simulation, and integrated optimization process. Because the volute size is large, the number of FEM and acoustical mesh is huge, and an optimized cycling takes 24-26 hours. However, the design variables change often causing, problems in a single calculation process during the optimization process. In the optimum iterative procedure, the optimization process terminates due to the failure of a single simulation program. Therefore, the long time of the optimal iterative procedure and the iterative procedure failure are the major bottlenecks in the optimization of acoustic performance for all complex structures. To resolve these bottlenecks, many scholars and engineers use a high-efficiency and high-precision approximate model instead of a complex simulation model to obtain the expected results. Therefore, a combination of a design of experiment (DOE) and an approximation model (AM) was used to determine the relationship between the structural design variables and the target function, and provided the basic data and models for the subsequent optimization. According to the studied centrifugal fan volute, taking each volute thickness (FT, BT, and ST) as design variables, each design variable was given five levels. Table 3 provide the levels of distribution of the design variables. Because of the smaller number of design variables, the full-factor method was adopted to collect sample points of the design space constructed by the three variables collected; thus, a total of 125 sample points was collected.

Table 3 The levels of distribution of each volute panel thickness

\begin{tabular}{ccccccc}
\hline variables & range $/ \mathrm{mm}$ & \multicolumn{5}{c}{ Levels of distribution $/ \mathrm{mm}$} \\
\hline FT & $5.00 \sim 10.0$ & 5.00 & 6.25 & 7.50 & 8.75 & 10.0 \\
ST & $5.00 \sim 10.0$ & 5.00 & 6.25 & 7.50 & 8.75 & 10.0 \\
BT & $5.00 \sim 10.0$ & 5.00 & 6.25 & 7.50 & 8.75 & 10.0 \\
\hline
\end{tabular}

For each sample point, the aforementioned vibro-acoustic coupling assessment method was used to calculate the radiated sound power and the total volute mass of the volute structure surface of each combination. The approximate model approach is generally divided into two broad categories: first, least squares fitting, also known as response surface methodology (RSM); and the second, interpolation. RSM uses polynomial functions to fit the design space. In addition, the RSM fitted the complex response relationships through regression models due to the simple algebraic expression. In addition, because of the advantages of being systematic and 
practical, the RSM had been used in a broad range [33-37], but the approaching effect is not as good as that of neural network and other methods for highly complex functional relationships. Radial basis function (RBF) belongs to the interpolation algorithms and is the second approximate model method. Because of the neural network model has a strong ability to approximate complex nonlinear functions, the learning speed is fast, it has excellent generalization ability, and it is highly fault-tolerant. It is used by many scholars and engineers in aircraft wingtips [38], white bodies [39], space vehicles [40], turbofan engines [41], compressors [42], ships [43], MMES controllers [44], and many other applications. The relationships between vibrational sound radiation of the volute casing surface and the volute panel thickness are typically non-linear. Thus, this study used the RBF model to approximate and fit the design space.

Figure 19 shows the fitting procedure of the vibro-acoustic optimization of volute casing. It can be seen from the figure that this procedure is mainly divided into two parts: the first is creation of a design space for the collected sample points using the assessment method of vibro-acoustic coupling. The FEM model of fan volute was established by UG, then ANSA constructed FEM mesh and the Nastran code solved the volute modal participation factor. At last, the vibrational sound radiation of volute was used by LMS Virtual Acoustics. In addition, all the calculation codes were integrated into the multi-disciplinary optimization platform Isight. The second built an RBF approximation model (RBF surrogate model) instead of a simulation loop as mentioned for the first part. Thus, the RBF method of Isight code was used to establish an approximate alternative model.

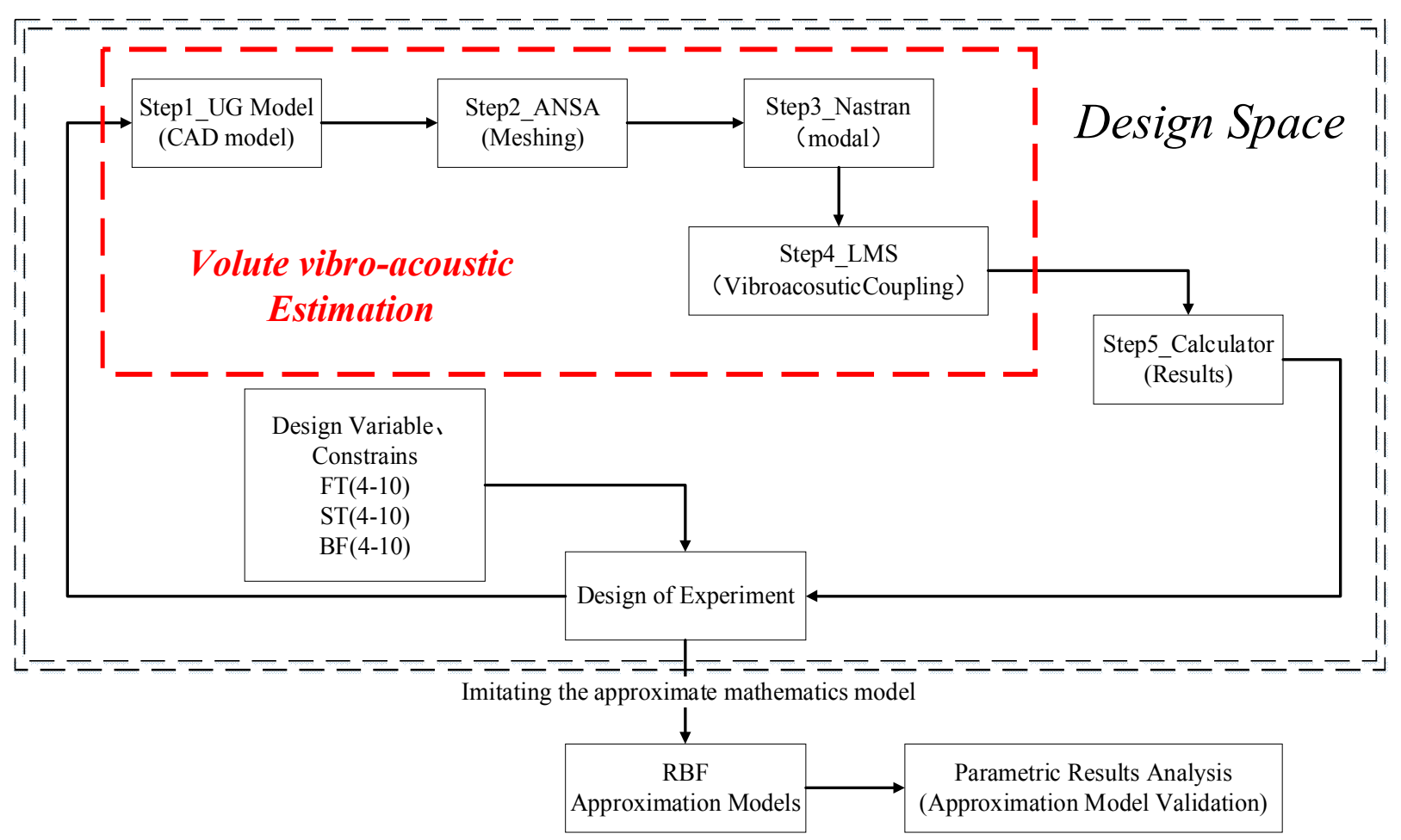

Figure19 Design space and RBF approximation model processing for optimization

In fact, the approximate model can be considered as an approximate approaching for the real response function, and the analysis of the approximate model is equal to the analysis of the real system. The precision of the approximate model is affected by the number of sampling points. At present, statistical theory with analysis of variance is usually used to verify the effectiveness of an approximate model. However, the approximate model is usually tested by the complex correlation coefficient $\mathrm{R}^{2}\left(0<\mathrm{R}^{2}<1\right)$ in engineering, and Shi [45] provides its mathematical expression. The closer the value of $\mathrm{R}^{2}$ is to 1 , the more precise the approximation model is. Figure 20 shows the error schematic diagram of each response surface model. In the figure, the Kirchhoff SPW (dB) represents the radiated sound power of the volute surface, and the mass $(\mathrm{kg})$ represents the total mass of the volute. It can be seen that all the response surface models are infinitely close to 1; thus, the approximate model established using the mentioned method can completely replace the real simulation loop. 


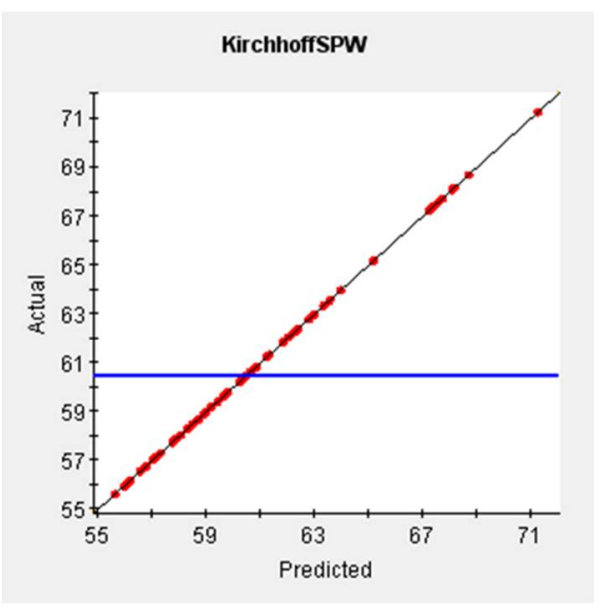

(a)

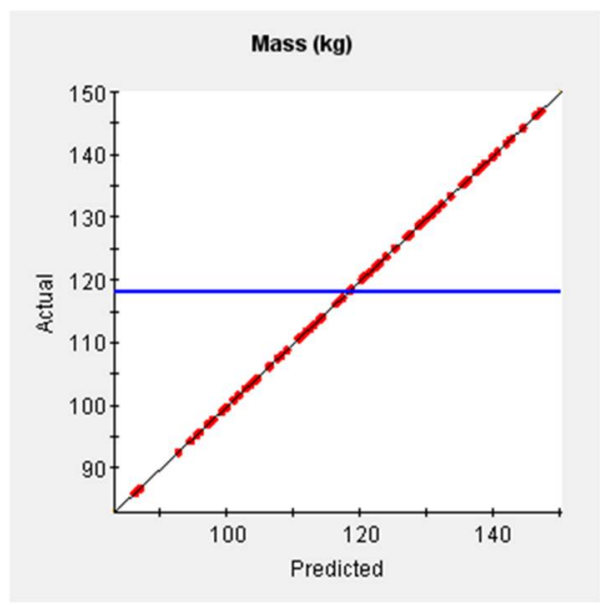

(b)

Figure 20 the error schematic diagram of each response surface model

Figure 21 shows the correlation distribution of design variables and responses (Kirchhoff SPW, volute mass on the volute structure surface), the correlation theory, and definition of major effect referred to in reference [46]. In addition, the positive value indicates that the target response is proportional to the design variable, and otherwise is an inverse relationship. The closer the absolute value of the coefficient is to 1 , the higher the degree of correlation. It can be seen from the figure that ST had the highest correlation with all the responses, followed by FT, then BT. Concerning radiated sound power, ST and FT present an inverse proportionality to the radiated sound power, which means that the larger the volute panel thickness (ST and FT), the smaller the radiated sound power. However, the main effect diagram of each volute panel (ST, BT, FT) in Fig. 22 shows that ST and FT are not in proportion to the acoustic power of the volute surface; a most reasonable range of ST and FT exists. The aforementioned analysis shows that ST is most sensitive to the radiated sound power of the volute structure surface, followed by BT, then FT. However, the thickness of each volute panel (ST, BT, and FT) is in direct proportion to the volute total mass, which means that the thicker the thickness of each volute panel, the greater the total mass of the casing.

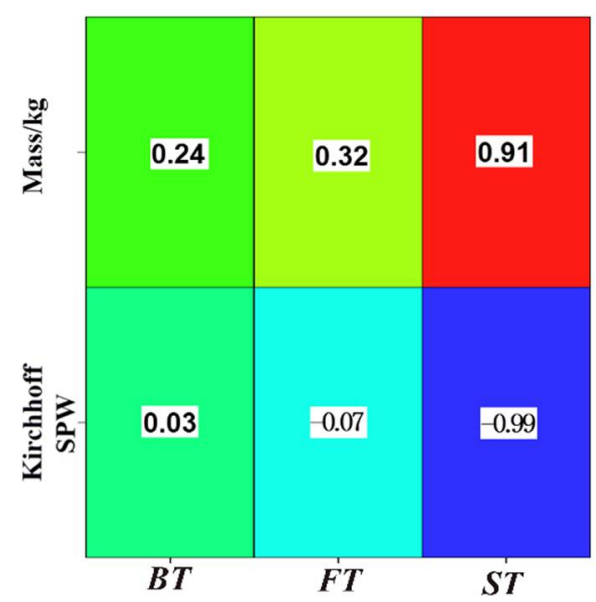

Figure 21 the correlation distribution of design variables and responses (Kirchhoff SPW and volute mass on the volute structure surface) 


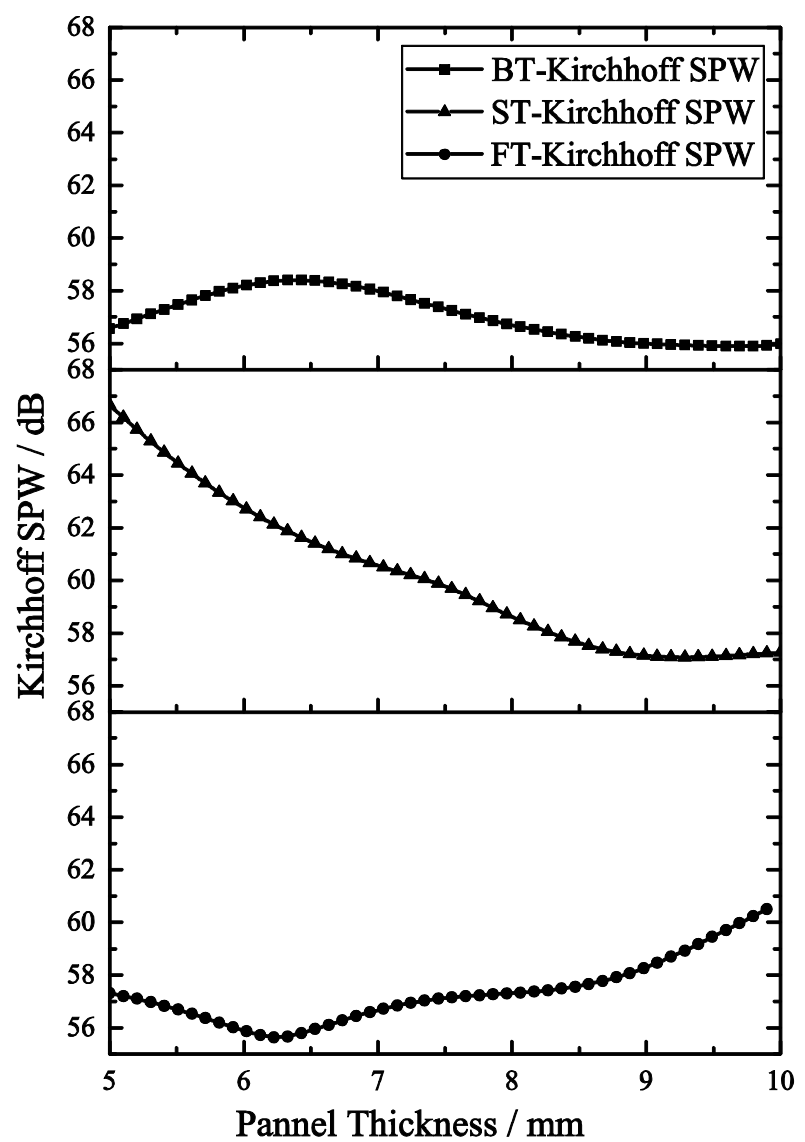

(a) the relationship of volute panel thickness and Kirchhoff SPW

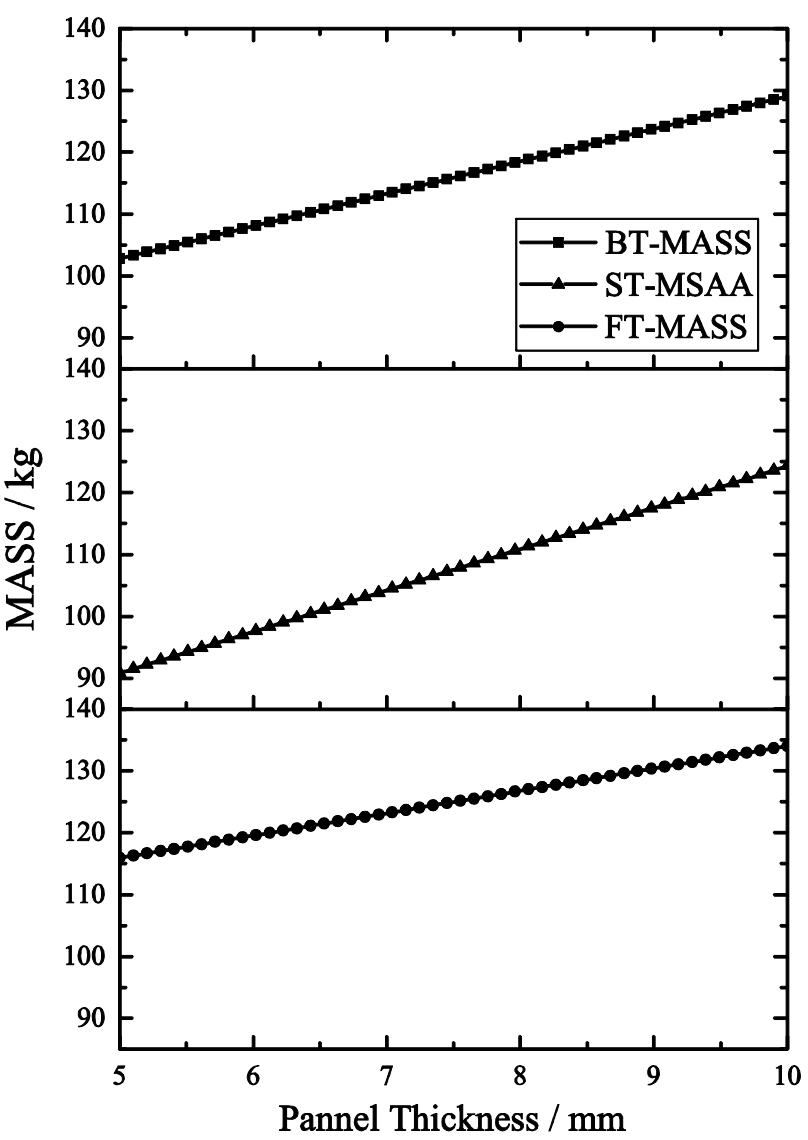

(b) the relationship of volute panel thickness and total mass

Figure 22 The main effect profile of the Kirchhoff SPW and the total mass of the volute

\subsection{Single-Objective Optimization}

The collection of sample points described in the previous text, based on the RBF approximation model, provides the basic database and the analysis model of the target function for the optimization of the vibro-acoustic coupling of the volute structure. The analysis results between the design variables and the objective function show that the combination of different volute panel thicknesses has different structural radiated sound power values, and presents an optimal combination of one or several groups of reasonable volute panel thickness, making the radiated sound power on the volute structure surface a minimum. Therefore, the optimal or compliant volute thickness combinations can be found through mathematical optimization methods. In this study, the single-objective optimization took three panel thickness (FT, BT, and ST in mm) as design variables, and took the vibrational sound radiated power of volute surface (Kirchhoff SPW (dB)) as the target function. In addition to these, the single-objective optimization was divided into two parts; the first part maintained the volute total mass as invariable, and for the second part, there was no mass constraint on the volute. Because the thickness of the volute panel is generally less than $10 \mathrm{~mm}$, the author assigned the volute panel thickness as from 4 to $10 \mathrm{~mm}$. Therefore, the mathematical model optimized in this section is as follows:

The objective function, $W_{s}$ (minimum):

$W_{s}=\sum_{\mathrm{j}=1}^{N_{e}} W_{j}=\frac{1}{2} \operatorname{Re}\left(\sum_{\mathrm{j}=1}^{N_{e}} \frac{1}{2} \int_{S_{j}} p_{j} v_{n j}^{*} d S\right),($ vibrational sound radiated power of volute surface, $\mathrm{W})$

Design viriables: $[\mathrm{FT}, \mathrm{ST}, \mathrm{BT}]$

$$
4 \leq \mathrm{FT} \leq 10
$$

Constrints: $4 \leq \mathrm{ST} \leq 10$

$$
4 \leq \mathrm{BT} \leq 10
$$


Constrained variable: $M_{T}=S_{F T} \cdot F T+S_{S T} \cdot S T+S_{B T} \cdot B T$, (total mass, $\mathrm{kg}$ )

Defining the sound power level as follow:

$10 * \lg \left(W_{s} / W_{r e f}\right)$

In the formula, $W_{r e f}$ represents the reference value of sound power, $W_{r e f}=1 \times 10^{-12} \mathrm{~W}$ 。

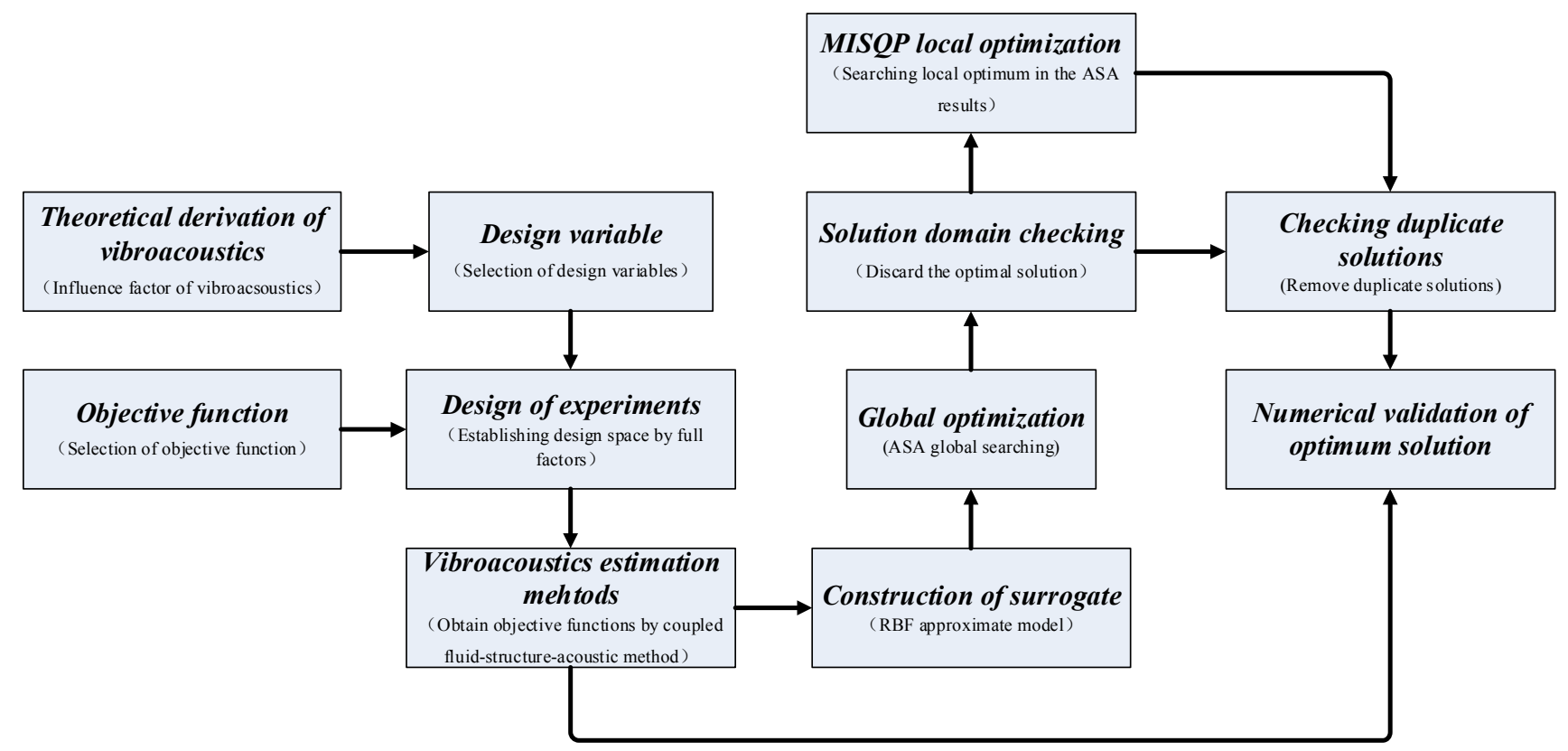

Figure 23 the flow chart of the single-objective optimization

Figure 23 shows the flow chart of the single-objective optimization. The single objective optimization uses the simulated annealing algorithm (ASA) to implement a global search. The optimal result of the approximate model in the previous text is assigned as the initial value, and the global optimization iterates 10,000 steps and takes 12 minutes. Then, the value is locally optimized using hybrid integer sequential quadratic programming (MISQP), which iterates more than 12 steps in several seconds. Table 4 shows the massless constrained optimization results. It can be seen that the radiated sound power on the volute structure surface decreases by $9.4 \mathrm{~dB}$. When ST increases to a maximum, the BT and ST locate at minimum values; unfortunately, the volute total mass increases by $18.13 \%$. Table 5 shows the optimization results with the volute total mass invariable. Even though the volute total mass remains the same, the radiated sound power on the surface of the volute will also be weakened by an average of $6.3 \mathrm{~dB}$. In addition, the radiated sound power spectrum of the volute structural surface shown in Fig. 24 also shows that the single objective optimization significantly improves the radiated sound power on the volute surface at the fundamental frequency (BPF).。

Table 4 the massless constrained optimization results

\begin{tabular}{ccllcc}
\hline & $\mathrm{FT}(\mathrm{mm})$ & $\mathrm{ST}(\mathrm{mm})$ & $\mathrm{BT}(\mathrm{mm})$ & $W_{\mathrm{s}} /(\mathrm{dB})$ & $\mathrm{M}_{\mathrm{T}}(\mathrm{kg})$ \\
\hline Original & 6.000 & 5.000 & 6.000 & 63.78 & 86.592 \\
Optimized & $\mathbf{4 . 0 0 0}$ & $\mathbf{1 0 . 0 0}$ & $\mathbf{4 . 0 0 0}$ & $\mathbf{5 4 . 3 8}$ & $\mathbf{1 0 2 . 2 9}$ \\
Numerical validated & $\mathbf{4 . 0 0 0}$ & $\mathbf{1 0 . 0 0}$ & $\mathbf{4 . 0 0 0}$ & $\mathbf{5 6 . 2 5}$ & $\mathbf{1 0 2 . 2 9}$ \\
\hline
\end{tabular}

Table 5 the optimization results with the volute total mass invariable

\begin{tabular}{cccccc}
\hline & $\mathrm{FT}(\mathrm{mm})$ & $\mathrm{ST}(\mathrm{mm})$ & $\mathrm{BT}(\mathrm{mm})$ & $W_{\mathrm{s}} /(\mathrm{dB})$ & $\mathrm{M}_{\mathrm{T}}(\mathrm{kg})$ \\
\hline Original & 6.000 & 5.000 & 6.000 & 63.78 & 86.592 \\
Optimized & $\mathbf{4 . 3 3 5}$ & $\mathbf{7 . 4 7 0}$ & $\mathbf{4 . 0 0 0}$ & $\mathbf{5 7 . 5 2}$ & $\mathbf{8 6 . 5 9 2}$
\end{tabular}




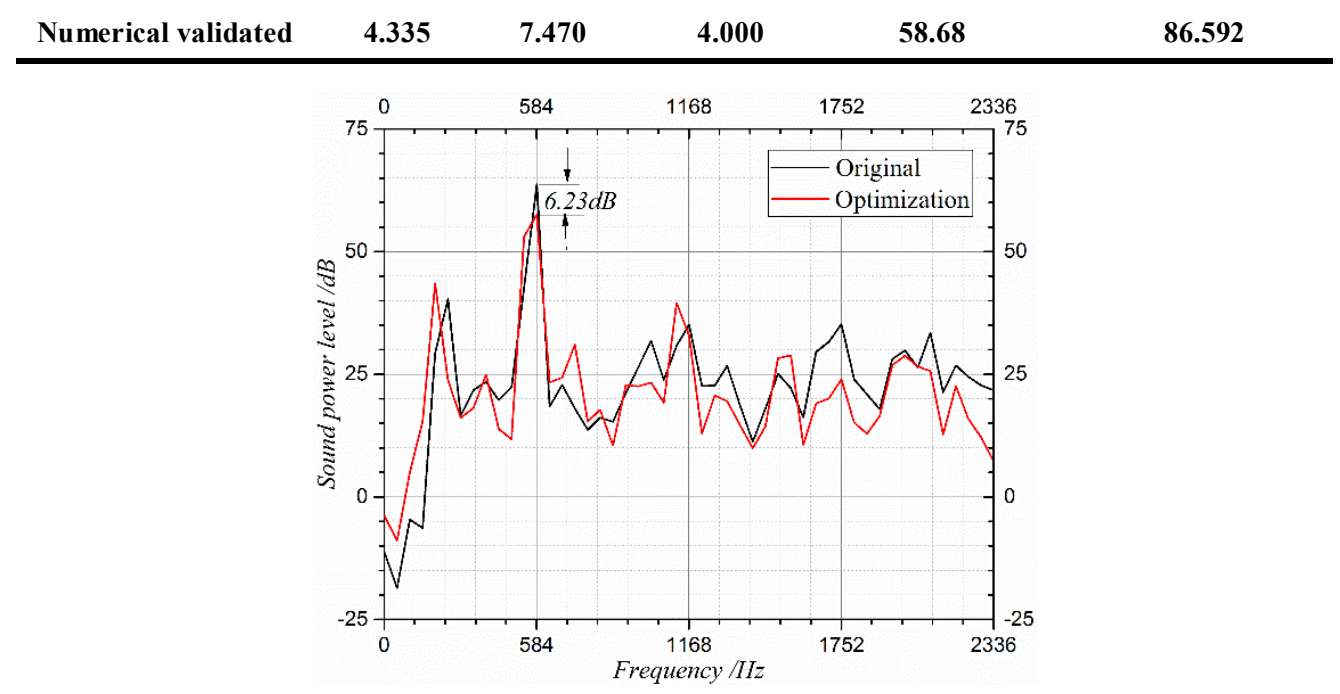

Figure 24 the radiated sound power spectrum of the volute structural surface

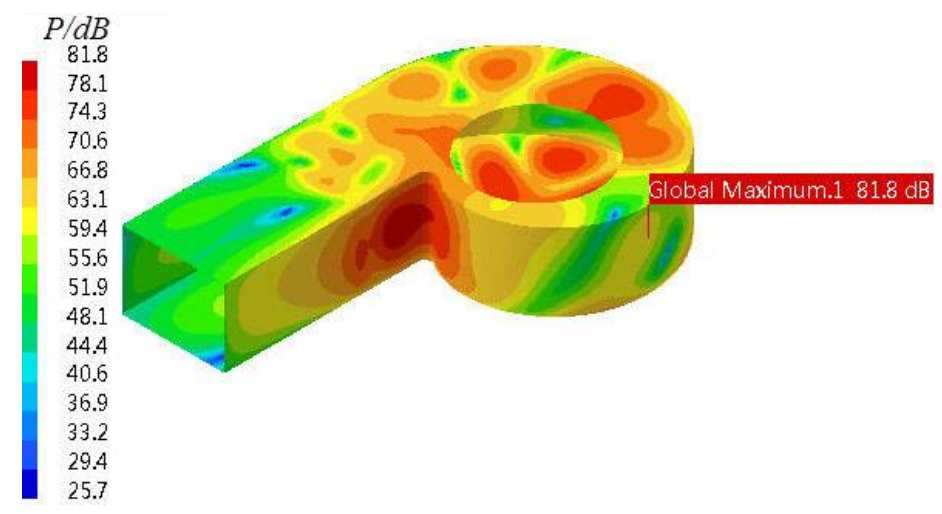

(a) the whole volute body

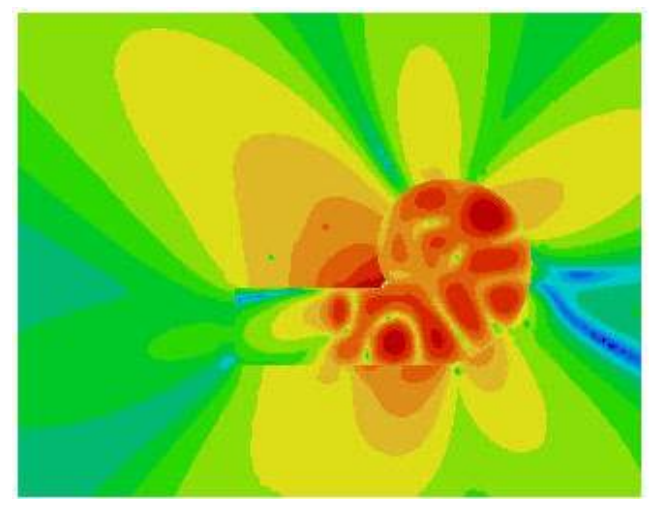

(b) XY section

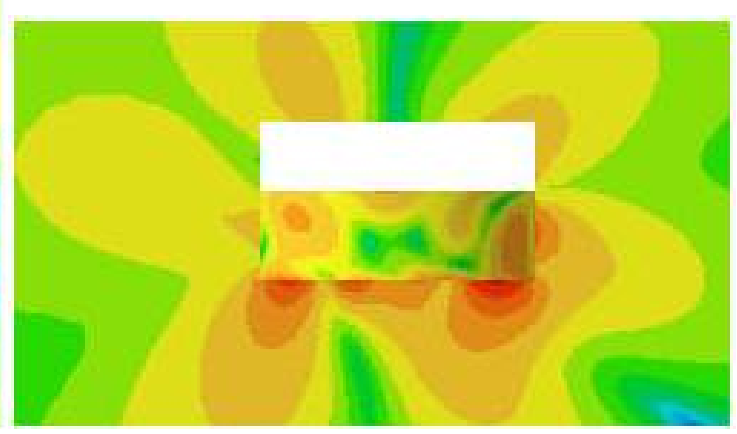

(c) ZY section

图 25 The distribution of vibrational sound radiation of original volute at the fundamental frequency

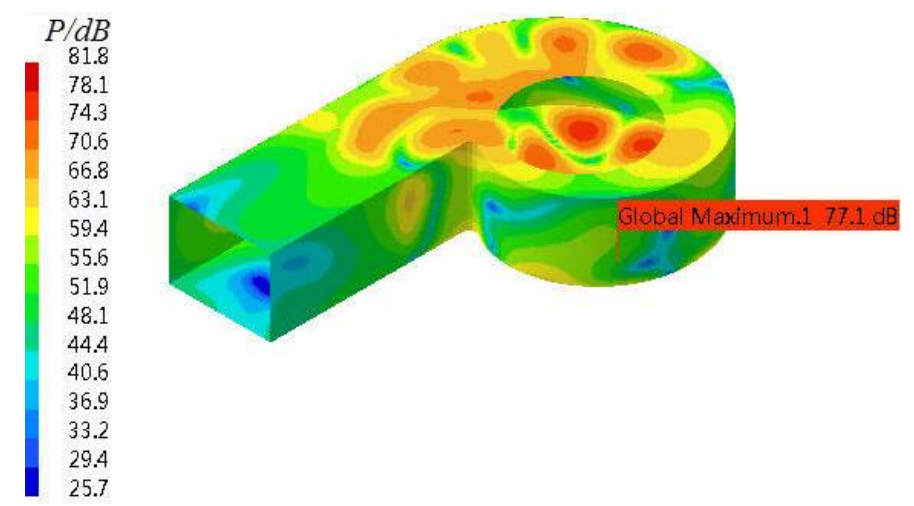

(a) the whole volute body 


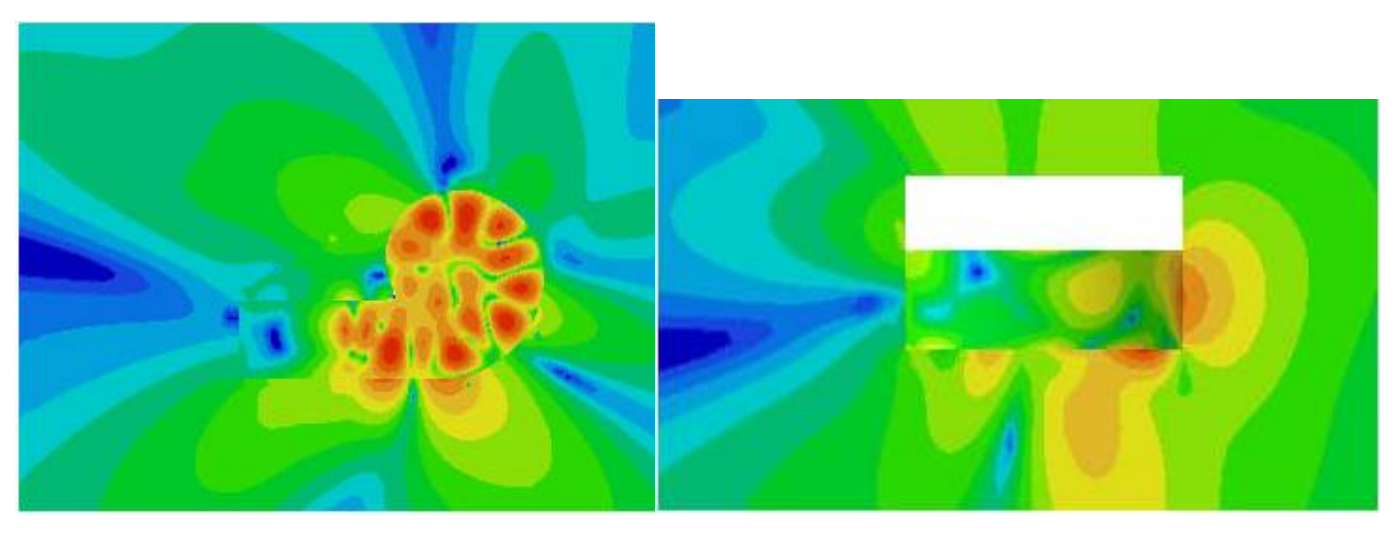

(b) XY section

(c) ZY section

Figure 26 The distribution of vibrational sound radiation after the total mass invariable optimization at the fundamental frequency

From the comparison of the distribution of vibrational sound radiation at the fundamental frequency and the different cross sections in Fig. 25 and Fig. 26, it can be concluded that the sound pressure of the vibrational radiation close to the tongue on the side volute is greatly reduced by optimization, and the other sound pressure of the vibrational radiation on the other area is also weakened to varying degrees. In addition to these results, the optimization also changed the directivity distribution of acoustical radiation, and very strong directivity was produced on the back panel side of the volute.

\section{3 multi-objective optimization}

Parametric analysis results show that the parameters of the vibrational sound power and total mass on the volute surface are somewhat contradictory. Thus, a multi-objective optimization method is needed to obtain the optimal volute thickness combination. The definition of the multi-objective optimization mathematical model is presented as follows:

The objective function, $W_{s}, \mathrm{M}_{\mathrm{T}}$ (minimize)

$$
\begin{aligned}
& W_{s}=\sum_{\mathrm{j}=1}^{N_{e}} W_{j}=\frac{1}{2} \operatorname{Re}\left(\sum_{\mathrm{j}=1}^{N_{e}} \frac{1}{2} \int_{S_{j}} p_{j} v_{n j}^{*} d S\right),(\text { vibrational sound radiated power of volute surface }, \mathrm{W}) \\
& M_{T}=S_{F \mathrm{~T}} \cdot F T+S_{S T} \cdot S T+S_{B T} \cdot B T,(\text { total mass, } \mathrm{kg})
\end{aligned}
$$

Design viriables: [FT, ST, BT]

$$
4 \leq \mathrm{FT} \leq 10
$$

Constrints: $4 \leq \mathrm{ST} \leq 10$

$$
4 \leq \mathrm{BT} \leq 10
$$




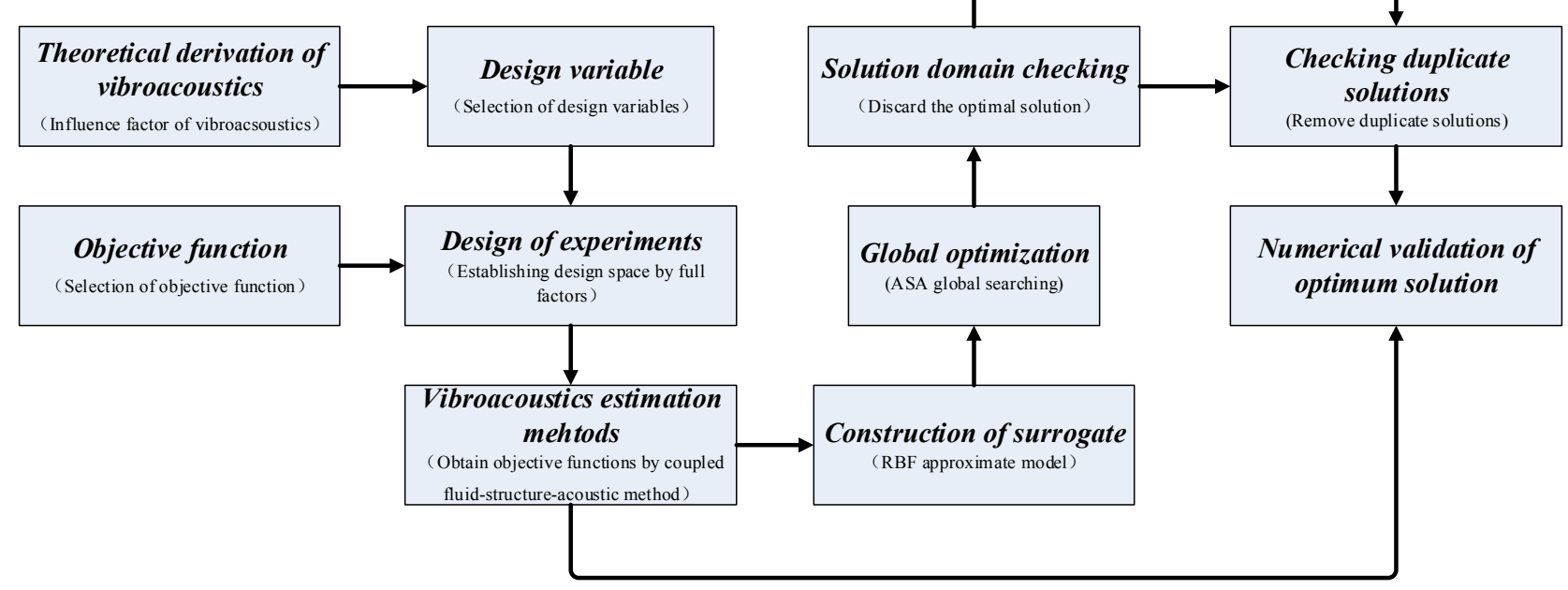

Figure 27 The flow chart of multi-objective optimization

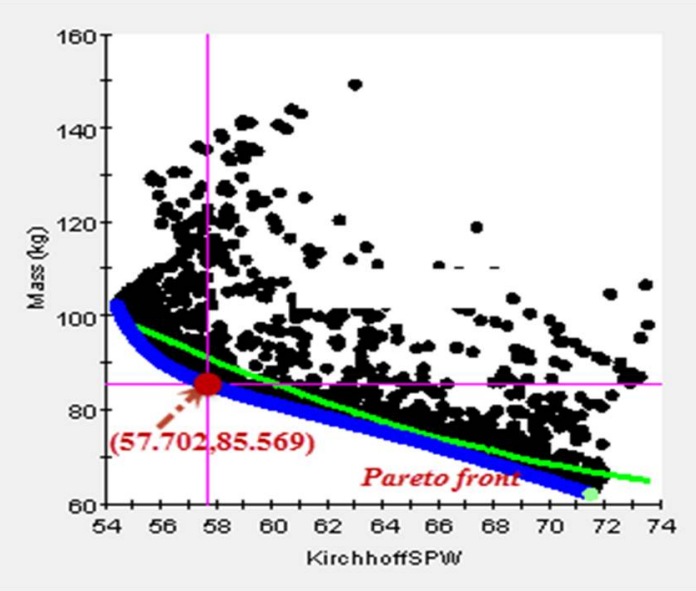

Figure 28 the distribution of Pareto front solutions

The multi-objective genetic algorithm termed NSGA_2 was adopted to solve the multi-objective optimization with the optimal value obtained by taking the single-objective optimization of specific mass constraints as initial values. Fig. 27 presents a flow chart of multi-objective optimization. The global search iterates 4800 steps and the Pareto frontier solutions are marked. A solution satisfying the requirement is selected as the initial value of MISQP for mixed integer quadratic programming, then the final solution is obtained by iterating 15 steps again. Finally, the unidirectional fluid-structure-acoustic coupling method is used to verify the precision of the optimal solution.

Table 6 The multi-objective optimization results

\begin{tabular}{cllccc}
\hline & $\mathrm{FT}(\mathrm{mm})$ & $\mathrm{ST}(\mathrm{mm})$ & $\mathrm{BT}(\mathrm{mm})$ & $W_{\mathrm{s}} /(\mathrm{dB})$ & $\mathrm{M}_{\mathrm{T}}(\mathrm{kg})$ \\
\hline Original & 6.000 & 5.000 & 6.000 & 63.78 & 86.592 \\
Optimized & $\mathbf{4 . 0 0 1}$ & $\mathbf{7 . 9 3 5}$ & $\mathbf{4 . 0 1 0}$ & $\mathbf{5 6 . 5 3}$ & $\mathbf{8 8 . 5 4 9}$ \\
Numerical validated & $\mathbf{4 . 0 0 1}$ & $\mathbf{7 . 9 3 5}$ & $\mathbf{4 . 0 1 0}$ & $\mathbf{5 7 . 1 6}$ & $\mathbf{8 8 . 5 4 9}$ \\
\hline
\end{tabular}




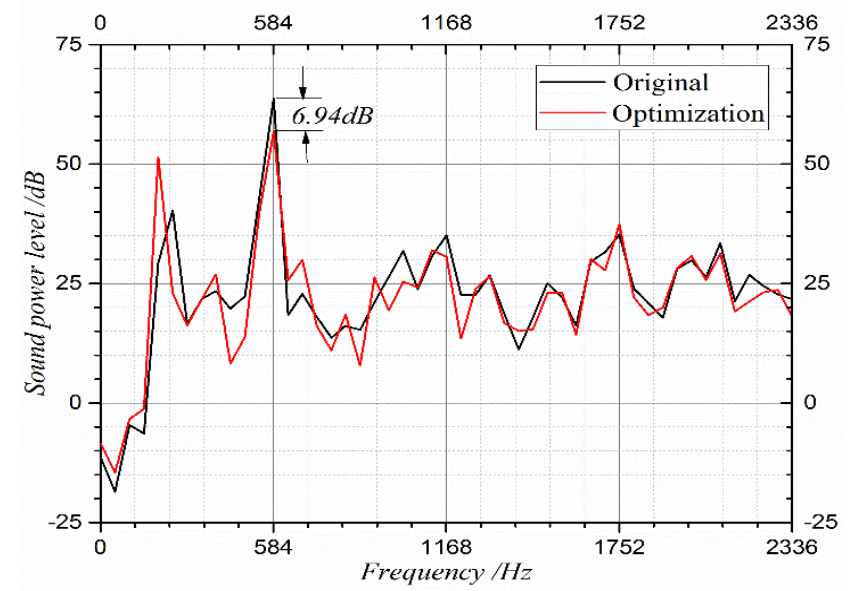

Figure 29 The frequency spectrum of total acoustic power of the volute surface

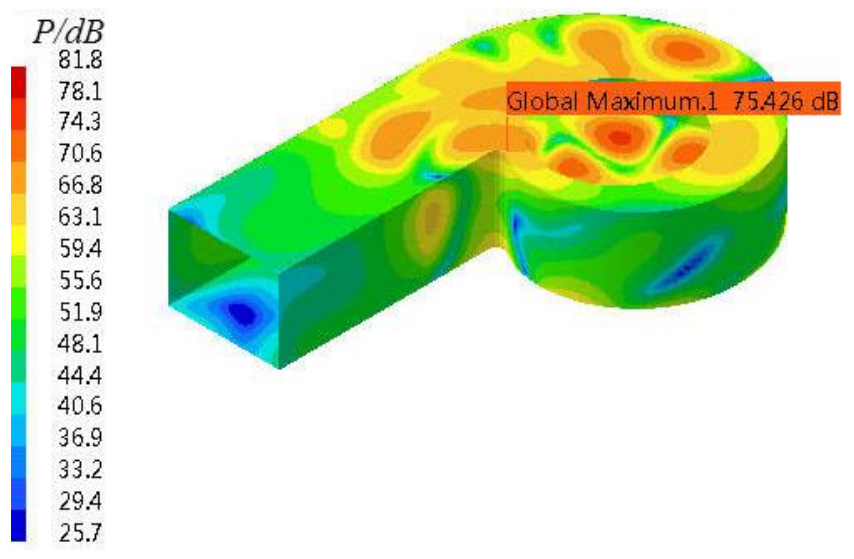

(a) the whole volute body

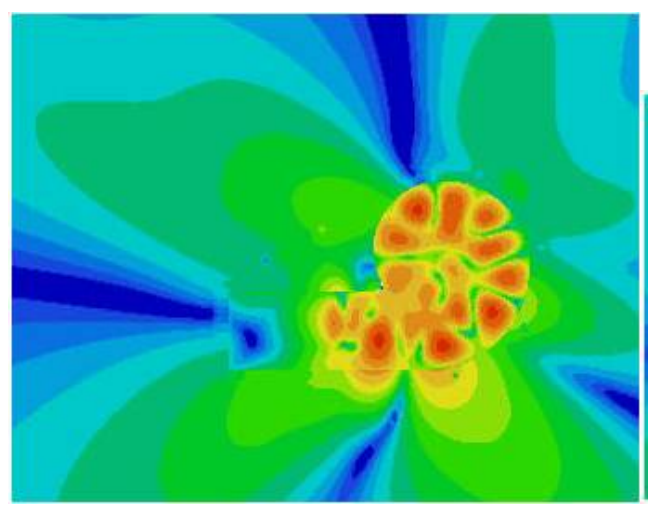

(b) XY section

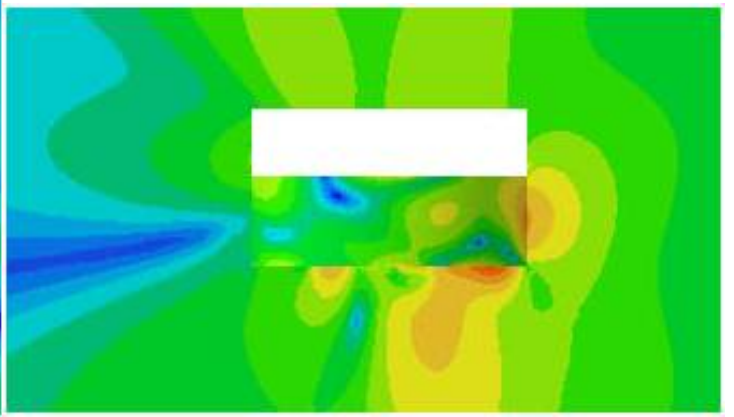

(c) ZY section

Figure 30 the radiated sound power distribution of the volute structure surface after multi-objective optimization

According to the distribution of Pareto front solutions in Fig. 28, it can be seen that the radiated sound power on the volute surface and the total mass of the volute show an approximately linear inverse proportional relationship, and a smaller vibrational sound power can be obtained, when the volute mass (mass increase control: $\pm 3 \mathrm{~kg}$ ) changes within a small range. Moreover, if the volute mass increased by $2 \mathrm{~kg}$, the total acoustic power of the volute surface and the fundamental radiated sound power decreased by $7.3 \mathrm{~dB}$ and $6.9 \mathrm{~dB}$, respectively, as shown in Table 6 and Fig. 29. In addition to these, Fig. 30 shows a comparison of the radiated sound power of the volute structure surface after multi-objective optimization. It can be seen that, after optimization, the multi-objective optimization presents the same noise reduction effect on the volute structure surface as the single-objective optimization. In addition to these, the radiation sound pressure close to the tongue on the volute front panel is greatly reduced, the sound pressure is lower (under $10 \mathrm{~dB}$ ) than at the other parts of the volute, and the sound pressure of the other volute parts is also greatly weakened. In summary, the radiated sound power of the volute structure surface obtained using the multi-objective optimization method is further reduced by nearly $1 \mathrm{~dB}$ compared to that of the single-objective optimization with mass constraints. It can be concluded that the optimization effect of the multi-objective method is obviously better than the single-objective optimization for the studied fan case. Even considering the two conflicting objective functions, the multi-objective optimization can achieve a more balanced effect. 


\section{Conclusions}

Concerning a fan system with long pipelines connected to the import and export, the external radiated noise is the component of structural vibration noise during fan operation, and is caused by the volute casing vibrations induced by the unsteady flow in the fan. Thus, to reduce this type of vibrational sound radiation, the main influencing factors of vibration noise on the volute surface were obtained through theoretical derivation. In this study, a vibrational noise control method of multi-disciplinary optimization which considered the influence of vibro-acoustic coupling was proposed. In this method, the panel thickness of the volute (FT, ST, and BT) was assigned as the design variable, and the sound radiated power of the volute surface and the volute total mass were set as target functions. Moreover, the DOE was applied to collect sampling points of the design sample, then an RBF approximating a surrogate model instead of an actual simulation loop was constructed. Finally, a single-objective and multi-objective optimization design was implemented. Thus, the main research conclusions are as follows:

(1) Regarding the problem of vibrational noise radiation, a one-way coupling method of fluid-structure-acoustic was implemented in this paper, and the effectiveness of this method was verified by dynamic pressure measurement and vibration measurement of the volute casing.

(2) The optimization results show that they do not follow the rule that the thicker each volute panel thickness, the lower the acoustical radiated power on the volute casing. Each volute panel thickness has an optimal combination. In addition to these results, sensitivity analysis showed that ST is the most sensitive to the acoustical radiated power of the structural surface, followed by BT, and then FT which is the smallest. Thus ST and BT should be the focus of subsequent optimization. However, FT, ST and BT are in direct proportion to total mass. Concerning optimization without mass constraint, the acoustical radiated power on the volute casing surface decreased by $9.4 \mathrm{~dB}$, but the volute total mass increased by $18.13 \%$. If the total mass of the volute was invariable, the acoustical radiated power decreased by $6.3 \mathrm{~dB}$. However, the acoustical radiated power on the volute surface and the total mass of the volute are two opposite parameters. Therefore, the multi-objective optimization was more advantageous. It was found that the volute acoustical radiated power on the volute surface decreased by $7.3 \mathrm{~dB}$, when the total mass of the volute slightly increased $( \pm 3 \mathrm{~kg})$. The optimization in this study provides an important technical reference for the design of a low vibro-acoustic volute.

Acknowledgements

The authors would like to acknowledge the financial supports given by National Natural Science Foundation of China (No.51236006) and Natural Science Basic Research Plan in Shaanxi Province of China (Program No. 2016JQ1043 and No. 2018JK0410).

\section{References}

[1] G.H. Koopmann, K.A. Cunefare, W. Neise, Fan casing noise radiation, Journal of Vibration \& Acoustics,113(1991)37-42.

[2] G.H. Koopmann, J.B. Fahnline. Designing quiet structures: a sound power minimization approach, Academic Press,1997.

[3] Won-Gul Hwang, Il-Kwon Oh, Byounggun Kim, Sungwoo Park, Kio Ryu. A study on noise radiation from compressor shell. International Compressor Engineering Conference. Purdue. 2006.

[4] Jiancheng Cai, Datong Qi, Fu-an Lu, et al. Numerical studys on fan casing vibration and noise radiation. Proceedings of ASME Turbo Expo, Orlando. USA. 2009.

[5] J.C. Cai, D.T. Qi, A quantitative study of the blade passing frequency noise fan. Journal of Vibration Engineering, 14(2012)12001211.

[6] J.C. Cai, D.T. Qi, F.A. Lu, X.F. Wen, Study of the Tonal Casing Noise of a Centrifugal Fan at the Blade Passing Frequency. Part II. Vibroacoustics, Journal of Low Frequency Noise Vibration \& Active Control, 29(2011)253-266.

[7] CAI Jian-cheng, QI Da-tong, TANG Yu, Quantitative prediction of sound radiation by casing vibration of centrifugal fan. Journal of Xi'an Jiao Tong University, 43(2009)71-74(In Chinese).

[8] Y.S. Kim, E.Y. Kim, S.K. Lee, Strategy for vibration reduction of a centrifugal turbo blower in a fuel cell electric vehicle based on vibrational power flow analysis, Proceedings of the Institution of Mechanical Engineers Part D Journal of Automobile Engineering,224(2010)985-995.

[9] HAN Bao-kun, ZHANG Wen-hao, CAO shu-ming, et al, Vibration and Noise Radiation Control of the Volute of Centrifugal Fan. Noise and Vibration Control, 2(2013)201-203. (In Chinese).

[10] S.J. Elliott, M.E. Johnson, Radiation modes and the active control of sound power,J Acoust Soc Am,94(1993)2194-2204.

[11] M.E. Johnson, S.J. Elliott, Active control of sound radiation using volume velocity cancellation, J Acoust Soc Am, 
98(1995)2174-2186.

[12] LI Shen, ZHAO De-you, Research on modal analysis of structural acoustic radiaton using structural vibration modes and acoustic radiation modes, ACTA Acustica, 29(2004)200-208. (In Chinese)

[13] YANG De-qing, LI Qing-dian, DAI Lang-tao, The optimal design of hydrazine bottle system to reduce vibration in satellite, Journal of Astronautics, 26(2005)804-807. (In Chinese)

[14] SHI Meng, ZHANG Jun, FANG Ji, et al, Study of Vibration and Noise Reduction by Minimizing Vibration Velocity for Plate Based on FEM/BEM, Journal of Da Lian Jiao Tong University, 30(2009)1-5. (In Chinese)

[15] ZHOU Zheng, LU Fu-an, QI Da-tong,et al, Numerical Optimization on Vibration and Noise Reduction of Centrifugal Fan Volute, Journal of Xi'an Jiao Tong University, 45(2011)59-64. (In Chinese)

[16] F.A. Lu, D.T. Qi, X.J. Wang, Z. Zhou, H.H. Zhou, A numerical optimization on the vibroacoustics of a centrifugal fan volute, Journal of Sound \& Vibration,331(2012)2365-2385.

[17] Nielsen, J C O, Acoustic optimization of railway sleepers, Journal of Sound and Vibration, 231(2000) $753-764$.

[18] L.Y. Chen, D.Y. Wang, Structural-acoustic optimization of stiffened panels based on a genetic algorithm, Journal of Marine Science \& Application,6(2007)55-61.

[19] J. Zhang, Z.W. Zhong, X.S. Ming, Z.W. Ying, Acoustic design optimization for coupled acoustic-structural systems, Journal of Vibration Engineering, 18(2005)519-523.

[20] E. Yuksel, G. Kamci, I. Basdogan, Vibro-Acoustic Design Optimization Study to Improve the Sound Pressure Level Inside the Passenger Cabin, Journal of Vibration \& Acoustics, 134(2012)61017.

[21] GJB 4058-2000, Noise and vibration measurement methods for equipments of naval ships. Beijing: China Commission of Science Technology and Industry for National Defense, 2000,

[22] GB/T 1236-2000, Industrial fan performance testing using standardized airway[S]. Beijing: Standards Press of China, 2001.

[23] J. Zhang, W. Chu, H. Zhang, Y. Wu, X. Dong, Numerical and experimental investigations of the unsteady aerodynamics and aero-acoustics characteristics of a backward curved blade centrifugal fan,Appl Acoust,110(2016)256-267.

[24] R. Ballesteros-Tajadura, S. Velarde-SuáRez, J.P. Hurtado-Cruz, Noise Prediction of a Centrifugal Fan: Numerical Results and Experimental Validation, Journal of Fluids Engineering,130(2008)253-257.

[25] J. Cai, D. Qi, F. Lu,Study of the tonal noise of a centrifugal fan at the blade passing frequency. Part I. Vibroacoustics, Journal of Low Frequency Noise, Vibration and Active Control, 29(2010) 253 266.

[26] Launder, E. B., Spalding, B. D., The numerical computation of turbulent flows, Computer Methods in Applied Mechanics \& Engineering, 3(1990)269-289.

[27] Y.Y. Jiang, S. Yoshimura, R. Imai, H. Katsura, T. Yoshida, C. Kato, Quantitative evaluation of flow-induced structural vibration and noise in turbomachinery by full-scale weakly coupled simulation, Journal of Fluids \& Structures,23(2007)531544.

[28] ISO 3744-1994, Acoustics-Determination of sound power levels of noise sources using sound pressure-Engineering method in an essentially free field over a reflecting plane [S].

[29] ZHANG Jian-hua, CHU Wu-li, DONG Xing-jie, et al, Numerical Investigation of Internal Fluid-induced Volute Vibration in a Marine Centrifugal Fan, Mechanical Science and Technology for Aerospace Engineering, 35(2016) 523-530. (In Chinese)

[30] Y. Zhang, W. Jiang, H. Wu, X. Zhou, J. Xu. A topology optimization design of compressor housing to reduce acoustic noise base on fast BEM[C]. 2013.

[31] S. Marburg, H.J. Hardtke, Efficient optimization of a noise transfer function by modification of a shell structure geometry Part II: Application to a vehicle dashboard, Structural \& Multidisciplinary Optimization,24(2002)60-71.

[32] M.S. Howe, Acoustics of Fluid-Structure Interactions, Phys Today, 52(1999)64.

[33] M.A. Nemeth, C.M. Borror, Response Surface Methodology: Process and Product Optimization Using Designed Experiments, 2nd edition (Book), J Qual Technol, (2003).

[34] R.H. Myers, D.C. Montgomery, G.G. Vining, C.M. Borror, S.M. Kowalski, Response Surface Methodology: A Retrospective and Literature Survey, J Qual Technol, 36(2004)53-78.

[35] LIU Xiao-liang, YUAN Min-jian, MIAO Yi-jun, et al, Numerical optimization of Volute outlet Structure for Forward-Curved Centrifugal Fan, Journal of Xi' an Jiao Tong University, 43(2009)61-65.(In Chinese).

[36] YU Hu, HOU Hong, DAI Yang, Optimization design of vibration response of range hood's shell, Journal of Vibration and Shock, 30(2011)179-182. (In Chinese). 
[37] M. Zangeneh, F. Mendonça, Y. Hahn, J. Cofer. 3D Multi-Disciplinary Inverse Design Based Optimization of a Centrifugal Compressor Impeller [C]. 2014.

[38] BAI Jun-qiang, WANG Dan, HE Xiao-long, et al, Application of an Improved RBF Neural Network on Aircraft Winglet Optimization Design, Acta Aeronautica ET Astronautica Sinica, 35(2014) 1865-1873.

[39] LU Fang, Research on body-in-white lightweight on multidisciplinary design optimization [D], JiLin University, 2014. (In Chinese).

[40] ZHANG Rui, SUN Guo-qing, YANG Ze-shan, Near Space Vehicle Entry Trajectory Optimization Design Based on OPTIMUS, Spacecraft Engineering, 21(2012) 79-84. (In Chinese).

[41] YANG Hua, GUO Ying-qing, Double variable PID decoupling control of turbofan engine based on RBF neural network identification, Journal of Aerospace Power, 22(2007) 1391-1395. (In Chinese).

[42] PENG Jing-bo, XIE Shu-sheng, Compressor characteristic simulation based on RBF neural network, Journal of Propulsion Technology, 27(2006)30-32. (In Chinese).

[43] LIN Ye-jin, REN Guang, Fuzzy Controller for Ship Steering Based on RBF Networks and Genetic Algorithms, Shipbuilding of China, 44(2003)73-79. (In Chinese).

[44] YU Jian-li, Optimal Design for MMSE Controller Based on RBF Neural Network, Journal of System Simulation, 25(2013) 2723-2728. (In Chinese).

[45] F. Shi, Numerical Study of Pressure Fluctuations Caused by Impeller-Diffuser Interaction in a Diffuser Pump Stage, Trans.asme J.fluids Eng,123(2001)466-474.

[46] Isight 5.8 user guide (Book), Dassault Systèmes Simulia Corp., 2003. 